\title{
Directed random walk in adsorbed monolayer
}

\author{
O.Bénichou $^{1}$, A.M.Cazabat ${ }^{2}$, M.Moreau ${ }^{1}$ and G.Oshanin $^{1}$ \\ ${ }^{1}$ Laboratoire de Physique Théorique des Liquides (CNRS - UMR 7600), \\ Université Pierre et Marie Curie, 4 place Jussieu, 75252 Paris Cedex 05, France \\ ${ }^{2}$ Laboratoire de Physique de la Matière Condensée, Collège de France, 11 place \\ M.Berthelot, 75252 Paris Cedex 05, France
}

\begin{abstract}
We study the dynamics of a tracer particle, which performs a totally directed random walk in an adsorbed monolayer composed of mobile hard-core particles undergoing continuous exchanges with a vapour phase. In terms of a mean-field-type approach, based on the decoupling of the tracer-particle-particle correlation functions into the product of pairwise, tracer-particle correlations, we determine the density profiles of the monolayer particles, as seen from the stationary moving tracer, and calculate its terminal velocity, $V_{t r}$. In the general case the latter is determined implicitly, as the solution of a certain transcendental equation. In two extreme limits of slow and fast monolayer particles diffusion, we obtain explicit asymptotic forms of $V_{t r}$. We show next that the density profile in the monolayer is strongly inhomogeneous: In front of the stationary moving tracer the local density is higher than the average value, $\rho_{L}$, and approaches $\rho_{L}$ as an exponential function of the distance from the tracer. Past the tracer the local density is lower than $\rho_{L}$ and the approach to $\rho_{L}$ may proceed differently depending whether the particles number in the monolayer is not or is explicitly conserved. In the former case the approach is described by an exponential dependence with a different characteristic length, compared to the behavior in front of the tracer; in the latter case, the density tends to $\rho_{L}$ algebraically. The characteristic lengths and the amplitudes of the density relaxation functions are also determined explicitly.
\end{abstract}

Key words: Hard-core lattice gas, Langmuir adsorption/desorption model, biased tracer diffusion.

PACS numbers: 05.40-a, 66.30.Lw, 68.45.Da 


\section{Introduction}

When a gas or vapour is brought into contact with a clean solid surface, parts of its molecules will become reversibly attached to the surface in the form of an adsorbed layer. Knowledge of equilibrium and dynamical properties of such adsorbed layers is essential for many practical applications, including coating, gluing and lubrication.

Following the seminal work of Langmuir (see, e.g. Ref.[1]), the equilibrium properties of adsorbed layers have been extensively studied and a great number of important developments have been made. In particular, further analysis included more realistic forms of intermolecular interactions or allowed for the possibility of multilayer formation. As a result, different possible phase transformations have been predicted and different forms of adsorption isotherms have been found, which well explain available experimental data (see, e.g. Refs.还运).

Some effort has been also invested to the understanding of molecular diffusion in adsorbed layers, which has a strong impact on their global dynamical behavior. For instance, diffusional processes control the rates of spreading of molecular films on solid surfaces [4, 河, spontaneous or forced dewetting of monolayers [6 9] or island formation [10]. Here, some approximate analytical results have been obtained for both dynamics of an isolated adatom on a corrugated surface and collective diffusion, describing spreading of the macroscopic density fluctuations in interacting adsorbates being in contact with the vapour phase [11 14]. On the other hand, most of available studies of the tracer diffusion in adsorbed layers, which provides a useful information about their intrinsic viscosity, pertain to strictly two-dimensional models excluding the possibility of particles adsorption and desorption (see, e.g. Refs. [15, 20] and references therein). Except for Ref. 21], which studied driven tracer dynamics in a somewhat artificial one-dimensional model, analysis of the tracer diffusion in adsorbed monolayers undergoing exchanges with the vapour phase seems to be lacking at present.

In this paper we study the diffusion properties of a driven tracer in a two-dimensional adsorbed monolayer undergoing continuous exchanges with the vapour. The system we consider consists of (i) a solid substrate, which is modeled in a usual fashion as a regular square lattice of adsorption sites supporting at most a single occupancy, (ii) a monolayer of adsorbed, mobile hard-core particles in contact with a vapour and (iii) a single, hard-core tracer particle. Each monolayer particle moves randomly along the lattice by performing hopping motion on the vacant neighboring lattice sites. The monolayer particles undergo continuous exchanges with a vapour phase, i.e. may desorb from and adsorb onto the lattice with some prescribed rates dependent on the vapour pressure, temperature and the interactions with the solid substrate. On the contrary, the tracer particle is constrained to move on the two-dimensional lattice only, i.e. it can not desorb to the vapour, and it is subject to a constant external force $E$. Hence, the tracer performs a biased random

walk, which is constrained by the hard-core interactions with the monolayer particles, and always remains within the monolayer, probing its response on the internal perturbancy or, in other words, its frictional properties. Here we focus on the limit of sufficiently large external force, such that the tracer may move only in one direction, but not large enough to make it slide regardless of the surface corrugation. The results for the general case of arbitrary $E$, which allow us to deduce the analog of the Stokes formula for 2D adsorbed monolayers and to define the corresponding friction coefficient, will be presented elsewhere 22].

In terms of a mean-field-type approximation of Ref. 23], which is based on the decoupling of the tracer-particle-particle correlation functions into the product of pair-wise correlations, we 
define the stationary density profiles of the monolayer particles, as seen from the moving tracer, and we determine analytically the terminal velocity $V_{t r}$ of the tracer. We show that the adsorbed monolayer particles tend to accumulate in front of the driven tracer, creating a sort of a "traffic jam", which impedes its motion. Thus the density profile around the tracer is highly inhomogeneous: the local density of the monolayer particles in front of the tracer is higher than the average and approaches the average value as an exponential function of the distance from the tracer. The characteristic length and the amplitude of the density relaxation function are calculated explicitly. On the other hand, past the tracer the local density is lower than the average; we show that depending on the condition whether the number of particles in the monolayer is explicitly conserved or not, the local density past the tracer may tend to the average value either as an exponential or as an algebraic function of the distance, revealing in the latter case especially strong memory effects and strong correlations between the particle distribution in the monolayer and the tracer position.

Further on, we find that the terminal velocity of the tracer particle depends explicitly on the excess density in the "jammed" region in front of the tracer. This excess density, in turn, depends on the magnitude of the velocity, as well as on the rate of the adsorption/desorption processes and on the rate at which the particles can diffuse away of the tracer. The interplay between the jamming of the monolayer, produced by the tracer particle, and the rate of its homogenization due to diffusion and adsorption/desorption processes, manifests itself as a medium-induced frictional force exerted on the tracer, whose magnitude depends on the tracer velocity. As a consequence of such a non-linear coupling, in the general case, (i.e. for arbitrary adsorption/desorption rates and particles diffusion coefficient), $V_{t r}$ can be found only implicitly, as the solution of a certain non-linear equation relating $V_{t r}$ to the system parameters. This equation simplifies considerably in the limit of small or large particles diffusivity, in which two cases exlicit asymptotic expressions for the tracer velocity are obtained. We finally remark, that a qualitatively similar physical effect was predicted recently for a different model system involving a charged particle moving at a constant speed at a small distance above the surface of an incompressible, infinitely deep liquid. It has been shown in Refs. 24, 25], that the interactions between the moving particle and the fluid molecules induce an effective frictional force exerted on the particle, producing a local distortion of the liquid interface, - a bump, which travels together with the particle and increases effectively its mass. The mass of the bump, which is analogous to the jammed region appearing in our model, depends itself on the particle's velocity resulting in a non-linear coupling between the medium-induced frictional force exerted on the particle and its velocity [24,25].

The paper is structured as follows: In Section 2, we formulate the model and introduce basic notations. In Section 3, we write down the dynamical equations which govern the time evolution of the monolayer particles and of the tracer. Section 4 is devoted to the analytical solution of these evolution equations in the limit $t \rightarrow \infty$; here we also present some general results on the form of the density profiles around stationary moving tracer and on the tracer terminal velocity, which is given implicitly, as the solution of a transcendental equation. In Section 5, we derive explicit asymptotic results for the tracer terminal velocity in the limits of small and large particles diffusivities. As well, here we discuss the forms of the density profiles at intermediate scales. Corresponding asymptotical behavior of the density profiles at large distances from the tracer is discussed in Section 6. Finally, we conclude in Section 7 with a brief summary and discussion of our results. 


\section{The model}

Consider a two-dimensional square lattice of adsorption sites $(X, Y)$, which is brought in contact with a reservoir of identic, electrically neutral particles (vapour phase) (Fig.1), maintained at a constant pressure. We suppose that the particles may leave the reservoir and adsorb onto any vacant lattice site at a fixed rate $f / \tau^{*}$. Then, the adsorbed particles may move randomly on the lattice by hopping with a rate $l / 4 \tau^{*}$ to any of four neighboring lattice sites, which process is constrained by hard-core exclusion preventing multiple occupancy of any site. They can also desorb from the lattice back to the reservoir at a site- and environment-independent rate $g / \tau^{*}$. We assume, for simplicity of exposition, that typical adsorption, desorption and jump times are equal to each other and are denoted by $\tau^{*}$. Consequently, at any time instant the variable $\eta(X, Y)$, characterizing the occupation of the site $(X, Y)$ such that

$$
\eta(X, Y)=\left\{\begin{array}{l}
1, \text { if the site }(X, Y) \text { is occupied by an adsorbed particle } \\
0, \text { if the site }(X, Y) \text { is empty, }
\end{array}\right.
$$

can change its value due to adsorption, desorption and constrained random hopping events. Note that the total number of particles in the adsorbed monolayer is not conserved in this dynamical process. However, the mean density $\rho_{L}$ of the adsorbate, $\rho_{L}=\langle\eta(X, Y)\rangle$, approaches as $t \rightarrow \infty$ a constant value

$$
\rho_{L}=\frac{f}{f+g}
$$

which equation is usually referred to as the Langmuir adsorption isotherm [1].

Further on, at $t=0$ we introduce at the lattice origin an extra hard-core particle, whose motion we would like to follow and which will be called in the remainder as the tracer. We stipulate that the tracer is different from the adsorbed particles in that it can not desorb from the lattice and in that it is subject to some external driving force, which favors its jumps to a preferential direction. Such a situation may be realized, for instance, if this only particle is charged and the system is subject to a uniform electric field. Here we will focus on the particular case when the external field, oriented in the positive $X$-direction, is sufficiently strong, such that the tracer performs totally directed random walk (Poisson process) along the $X$-axis in the two-dimensional monolayer of adsorbed mobile, hard-core particles. The tracer can be thought off as a certain external probe, which is designed to measure the resistance offered by the monolayer particles to the external perturbance.

More precisely, we define the tracer dynamics as follows: We suppose that the tracer, which is at the site $X_{t r}(t)$ at time $t$, waits an exponentially distributed time with mean? $\tau$, and then attempts to hop onto the neighboring site $X_{t r}(t)+\sigma, \sigma$ being the lattice spacing. The jump is actually fulfilled only if the target site is vacant at this moment of time; otherwise, i.e., if the

\footnotetext{
${ }^{1}$ These times, $\tau_{a d}, \tau_{d e s}$ and $\tau_{j u m p}$, respectively, can be readily made different and restored in our final results by the mere replacement $f \rightarrow \tau^{*} f / \tau_{a d}, g \rightarrow \tau^{*} g / \tau_{d e s}$ and $l \rightarrow l \tau^{*} / \tau_{\text {jump }}$.

${ }^{2}$ We suppose that in the general case this mean time $\tau$ is different from the corresponding time $\tau^{*}$ associated with the monolayer particles dynamics. As a matter of fact, this should be the case merely because the tracersubstrate interactions may be different from the particle-substrate ones. Varying $\tau$ we can mimic different possible situations; in particular, $\tau=0$ corresponds to the case when the tracer simply slides along the substrate regardless of the surface corrugation.
} 
target site is occupied by any adsorbed particle, the jump is rejected and the tracer remains at its position.

\section{Evolution equations}

Let $P\left(X_{t r}, \eta ; t\right)$ denote the probability of finding at time $t$ the tracer at the site $\left(X_{t r}, 0\right)$ and all adsorbed particles in the configuration $\eta=\{\eta(X, Y)\}$. Further more, let $\eta^{(X, Y) ;\left(X^{\prime}, Y^{\prime}\right)}$ denote the configuration obtained from $\eta$ by exchanging the occupation variables of the two neighboring sites $(X, Y)$ and $\left(X^{\prime}, Y^{\prime}\right)$, which describes the Kawasaki-type particle-vacancy exchange between the sites $(X, Y)$ and $\left(X^{\prime}, Y^{\prime}\right)$. Next, we denote as $\eta^{(X, Y)}$ the configuration obtained from the original configuration $\eta$ by the replacement $\eta(X, Y) \rightarrow 1-\eta(X, Y)$, which corresponds to the Glauber-type flip of the occupation variable due to the adsorption/desorption events.

Then, counting up all events which can result in the configuration $\left(X_{t r}, \eta\right)$ at time $t$ or modify it, we write down the following master equation, which governs the time evolution of the configuration probability $P\left(X_{t r}, \eta ; t\right)$ :

$$
\begin{aligned}
\dot{P}\left(X_{t r}, \eta ; t\right) & =\frac{l}{4 \tau^{*}} \sum_{(X, Y)} \prime \prime\left\{P\left(X_{t r}, \eta^{(X, Y) ;(X+\sigma, Y)} ; t\right)-P\left(X_{t r}, \eta ; t\right)\right\}+ \\
& +\frac{l}{4 \tau^{*}} \sum_{(X, Y)} \prime \prime\left\{P\left(X_{t r}, \eta^{(X, Y) ;(X, Y+\sigma)} ; t\right)-P\left(X_{t r}, \eta ; t\right)\right\}+ \\
& +\frac{1}{\tau}\left\{\left(1-\eta\left(X_{t r}, 0\right)\right) P\left(X_{t r}-\sigma, \eta ; t\right)-\left(1-\eta\left(X_{t r}+\sigma, 0\right)\right) P\left(X_{t r}, \eta ; t\right)\right\}+ \\
& +\frac{g}{\tau^{*}} \sum_{(X, Y)}^{\prime}\left\{(1-\eta(X, Y)) P\left(X_{t r}, \eta^{(X, Y)} ; t\right)-\eta(X, Y) P\left(X_{t r}, \eta ; t\right)\right\}+ \\
& +\frac{f}{\tau^{*}} \sum_{(X, Y)}^{\prime}\left\{\eta(X, Y) P\left(X_{t r}, \eta^{(X, Y)} ; t\right)-(1-\eta(X, Y)) P\left(X_{t r}, \eta ; t\right)\right\},
\end{aligned}
$$

where the dot denotes the time derivative, double prime indicates that the summation extends over all lattice sites $(X, Y)$, excluding the special sites $\left(X_{t r}-\sigma, 0\right)$ and $\left(X_{t r}, 0\right)$; a single prime after the summation sign means that the sums runs over all lattice sites excluding the site $\left(X_{t r}, 0\right)$ only.

Now, we can obtain the instantaneous velocity $V_{t r}(t)$ of the tracer particle directly from Eq.(2). To do this, we multiply both sides of Eq.(2) by $X_{t r}$ and sum over all possible configurations $\left(X_{t r}, \eta\right)$, which yields the following equation for the instantaneous tracer velocity

$$
V_{t r}(t)=\frac{d \overline{X_{t r}(t)}}{d t}=\frac{\sigma}{\tau}(1-k(\sigma, 0 ; t))
$$

where the bar denotes the configurational average and

$$
k\left(\lambda_{1}, \lambda_{2} ; t\right)=\sum_{\left(X_{t r}, \eta\right)} \eta\left(X_{t r}+\lambda_{1}, \lambda_{2}\right) P\left(X_{t r}, \eta ; t\right)
$$

is the probability of having at time $t$ an adsorbed particle at position $\left(\lambda_{1}, \lambda_{2}\right)$, defined in the frame of reference moving with the tracer particle. In other words, $k\left(\lambda_{1}, \lambda_{2}\right)$ can be interpreted as the density profile as seen from the moving tracer. 
Consequently, Eq.(3) relates the instantaneous velocity of the tracer particle to the particle density in the immediate vicinity of the tracer. Note, that if the monolayer was perfectly stirred, i.e. $k\left(\lambda_{1}, \lambda_{2}\right)=\rho_{L}$ everywhere, (which implies decoupling of $X_{t r}$ and $\eta$ ), we should obtain from Eq.(3) a trivial mean-field result

$$
V_{t r}=\frac{\sigma\left(1-\rho_{L}\right)}{\tau},
$$

which states that the tracer jump time $\tau$ is merely renormalized by a factor $\left(1-\rho_{L}\right)^{-1}$, which is the inverse concentration of voids in the monolayer: $\left(1-\rho_{L}\right) / \tau$ defines simply the frequency of successful jump events. However, this turns out not to be the case and $k\left(\lambda_{1}, \lambda_{2}\right)$ appears to be different of the equilibrium value $\rho_{L}$ everywhere, except for infinitely separated sites, $\lambda_{1,2} \rightarrow \pm \infty$. Moreover, as we proceed to show, $k\left(\lambda_{1}, \lambda_{2}\right)$ is itself dependent on the tracer velocity which yields ultimately a strongly non-linear equation determining $V_{t r}$.

Hence, in order to calculate the mean velocity of the tracer we have to determine the form of the density profile $k\left(\lambda_{1}, \lambda_{2}\right)$, or more precisely, the value of the mean density at the neighboring to the tracer site $\left(\lambda_{1}=\sigma, \lambda_{2}=0\right)$. The latter can be found from the master equation (2) by multiplying both sides of Eq.(2) by $\eta\left(X_{t r}+\lambda_{1} ; \lambda_{2}\right)$ and performing the summation over all configurations.

In doing so, we find the following set of equations, which hold for the sites separated from the tracer by the distance exceeding the lattice spacing $\sigma$, i.e. such that $\lambda_{1}^{2}+\lambda_{2}^{2}>\sigma^{2}$,

$$
\begin{array}{r}
\dot{k}\left(\lambda_{1}, \lambda_{2} ; t\right)=\frac{l}{4 \tau^{*}}\left\{\triangle_{\lambda_{1}}+\triangle_{\lambda_{2}}\right\} k\left(\lambda_{1}, \lambda_{2} ; t\right)-\frac{f+g}{\tau^{*}} k\left(\lambda_{1}, \lambda_{2} ; t\right)+\frac{f}{\tau^{*}}+ \\
+\frac{1}{\tau} \sum_{\left(X_{t r}, \eta\right)}\left(1-\eta\left(X_{t r}+\sigma, 0\right)\right) P\left(X_{t r}, \eta ; t\right)\left\{\eta\left(X_{t r}+\lambda_{1}+\sigma, \lambda_{2}\right)-\eta\left(X_{t r}+\lambda_{1}, \lambda_{2}\right)\right\},
\end{array}
$$

where the symbols $\triangle_{\lambda_{1,2}}$ denote the central second-order finite difference operators of step $\sigma$,

$$
\triangle_{\lambda_{1}} k\left(\lambda_{1}, \lambda_{2} ; t\right)=k\left(\lambda_{1}+\sigma, \lambda_{2} ; t\right)+k\left(\lambda_{1}-\sigma, \lambda_{2} ; t\right)-2 k\left(\lambda_{1}, \lambda_{2} ; t\right)
$$

On the other hand, for the sites adjacent to the tracer position, i.e. $(\sigma, 0),(-\sigma, 0)$ and $(0, \pm \sigma)$, respectively, we get

$$
\begin{aligned}
\dot{k}(\sigma, 0 ; t) & =\frac{l}{4 \tau^{*}}\left\{\nabla_{\lambda_{1}}+\triangle_{\lambda_{2}}\right\} k(\sigma, 0 ; t)-\frac{f+g}{\tau^{*}} k(\sigma, 0 ; t)+\frac{f}{\tau^{*}}+ \\
& +\frac{1}{\tau} \sum_{\left(X_{t r}, \eta\right)}\left(1-\eta\left(X_{t r}+\sigma, 0\right)\right) P\left(X_{t r}, \eta ; t\right) \eta\left(X_{t r}+2 \sigma, 0\right), \\
\dot{k}(-\sigma, 0 ; t) & =\frac{l}{4 \tau^{*}}\left\{\nabla_{\lambda_{1}}+\triangle_{\lambda_{2}}\right\} k(-\sigma, 0 ; t)-\frac{f+g}{\tau^{*}} k(-\sigma, 0 ; t)+\frac{f}{\tau^{*}}+ \\
& -\frac{1}{\tau} \sum_{\left(X_{t r}, \eta\right)}\left(1-\eta\left(X_{t r}+\sigma, 0\right)\right) P\left(X_{t r}, \eta ; t\right) \eta\left(X_{t r}-\sigma, 0\right),
\end{aligned}
$$

and

$$
\begin{gathered}
\dot{k}(0, \pm \sigma ; t)=\frac{l}{4 \tau^{*}}\left\{\nabla_{\lambda_{2}}+\triangle_{\lambda_{1}}\right\} k(0, \pm \sigma ; t)-\frac{f+g}{\tau^{*}} k(0, \pm \sigma ; t)+\frac{f}{\tau^{*}}+ \\
+\frac{1}{\tau} \sum_{\left(X_{t r}, \eta\right)}\left(1-\eta\left(X_{t r}+\sigma, 0\right)\right) P\left(X_{t r}, \eta ; t\right)\left\{\eta\left(X_{t r}+\sigma, \pm \sigma\right)-\eta\left(X_{t r}, \pm \sigma\right)\right\}
\end{gathered}
$$


where $\nabla_{\lambda_{1,2}}$ stand for the forward difference operators, of the form

$$
\nabla_{\lambda_{1}} k\left(\lambda_{1}, \lambda_{2} ; t\right)=k\left(\lambda_{1}+\sigma, \lambda_{2} ; t\right)-k\left(\lambda_{1}, \lambda_{2} ; t\right)
$$

and

$$
\nabla_{\lambda_{2}} k\left(\lambda_{1}, \lambda_{2} ; t\right)=k\left(\lambda_{1}, \lambda_{2}+\sigma ; t\right)-k\left(\lambda_{1}, \lambda_{2} ; t\right)
$$

Note, that Eqs.(8) to (10) are different from Eq.(6), since the density profile at the neighboring to the tracer sites is strongly perturbed by its asymmetric hopping rules. As a matter of fact, Eqs.(8) to (10) can be thought off as the boundary conditions for Eq.(6).

Now, several remarks on Eqs.(6) to (10) are in order. First of all, one notices that these equations are not closed with respect to $k\left(\lambda_{1}, \lambda_{2}\right)$ but rather coupled to the third-order, tracerparticle-particle correlation functions. In turn, if we attempt to derive the evolution equations for the third-order correlation functions, we find that the latter appear to be coupled to the fourthorder correlations. Consequently, in order to determine the tracer velocity, one faces the problem of solving an infinite hierarchy of coupled equations for the correlation functions. Here we resort to the simplest non-trivial closure of Eqs.(6) to (10) in terms of the pairwise correlation function $k\left(\lambda_{1}, \lambda_{2}\right)$, which is based on the following decoupling approximation of the third-order correlation functions,

$$
\begin{aligned}
& \sum_{\left(X_{t r}, \eta\right)} \eta\left(X_{t r}+\lambda_{1}, \lambda_{2}\right)\left(1-\eta\left(X_{t r}+\sigma, 0\right)\right) P\left(X_{t r}, \eta ; t\right) \approx \\
\approx & \left\{\sum_{\left(X_{t r}, \eta\right)} \eta\left(X_{t r}+\lambda_{1}, \lambda_{2}\right) P\left(X_{t r}, \eta ; t\right)\right\} \times\left\{\sum_{\left(X_{t r}, \eta\right)}\left(1-\eta\left(X_{t r}+\sigma, 0\right)\right) P\left(X_{t r}, \eta ; t\right)\right\}= \\
= & k\left(\lambda_{1}, \lambda_{2} ; t\right)(1-k(\sigma, 0 ; t)),
\end{aligned}
$$

i.e. the average with the weight $P\left(X_{t r}, \eta ; t\right)$ of the product of several occupation numbers of different sites is set equal to the product of their average values with the weight $P\left(X_{t r}, \eta ; t\right)$.

We hasten to remark that the approximate closure of the evolution equations in Eq. 13, as well as some similar but not obviously equivalent approximations 16, 17, have been already employed for studying related models of tracer diffusion in hard-core lattices gases and shown to provide quite accurate description of the dynamical and stationary behavior. The decoupling in Eq.(13) has been first introduced in Ref. 23] to determine the properties of the driven tracer diffusion in a one-dimensional hard-core lattice gas with the conserved number of particles, i.e. without exchanges of particles with the reservoir. Extensive numerical simulations performed in Ref.23. have demonstrated that such a decoupling is quite a plausible approximation for the model under study. Moreover, rigorous probabilistic analysis of Ref. [26] has shown that for this model the results based on the decoupling scheme in Eq.(13) are essentially exact. Furthermore, the same closure procedure has been applied recently to study spreading of a hard-core lattice gas from a reservoir attached to one of the lattice sites [5], and to treat the biased tracer dynamics in a onedimensional model of adsorbed monolayer in contact with a vapour phase, i.e. a one-dimensional version of the model to be studied here. Also in these cases an excellent agreement has been observed between the analytical predictions and Monte Carlo simulations data. Last but not least, as we set out to show elsewhere [22], in case of arbitrary force exerted on the tracer the 
decoupling in Eq.(13) reproduces the results of Refs. 116] and [17] for the tracer diffusion coefficient in two-dimensional hard-core lattice gases with a conserved particles number (the limit $f, g=0$, $f / g=$ const in our case); these results are known to be exact in the limits of small and large particle densities and provide a very accurate approximation for the tracer diffusion coefficient in two-dimensional hard-core lattice gases with arbitrary particle density [15]. We thus expect that it will render a plausible description of the tracer dynamics in the two-dimensional model under study and base our further analysis on this mean-field-type approximation.

Using the approximation in Eq.(13), we can rewrite Eq.(16) in the following closed form

$$
\dot{k}\left(\lambda_{1}, \lambda_{2} ; t\right)=\tilde{L} k\left(\lambda_{1}, \lambda_{2} ; t\right)+\frac{f}{\tau^{*}},
$$

where the operator $\tilde{L}$ is given by

$$
\tilde{L}=\frac{l}{4 \tau^{*}}\left\{\triangle_{\lambda_{1}}+\triangle_{\lambda_{2}}\right\}+\frac{1}{\tau}\{1-k(\sigma, 0 ; t)\} \nabla_{\lambda_{1}}-\frac{f+g}{\tau^{*}}
$$

Further more, we find

$$
\begin{gathered}
\dot{k}(\sigma, 0 ; t)=\left.\left(\tilde{L} k\left(\lambda_{1}, \lambda_{2} ; t\right)\right)\right|_{\lambda_{1}=\sigma, \lambda_{2}=0}+\frac{f}{\tau^{*}}+\frac{1}{\tau}\{1-k(\sigma, 0 ; t)\} k(\sigma, 0 ; t)+\frac{l}{4 \tau^{*}} k(\sigma, 0 ; t), \\
\dot{k}(-\sigma, 0 ; t)=\left.\left(\tilde{L} k\left(\lambda_{1}, \lambda_{2} ; t\right)\right)\right|_{\lambda_{1}=-\sigma, \lambda_{2}=0}+\frac{f}{\tau^{*}}+\frac{l}{4 \tau^{*}} k(-\sigma, 0 ; t),
\end{gathered}
$$

and

$$
\dot{k}(0, \pm \sigma, 0 ; t)=\left.\left(\tilde{L} k\left(\lambda_{1}, \lambda_{2} ; t\right)\right)\right|_{\lambda_{1}=0, \lambda_{2}= \pm \sigma}+\frac{f}{\tau^{*}}+\frac{l}{4 \tau^{*}} k(0, \pm \sigma ; t)
$$

Equations (14) to (18) constitute a closed system of equations, which suffice the computation of the density profiles and tracer velocity. Note, however, that these equation are non-linear, since $k\left(\lambda_{1}=\sigma, 0\right)$ enters the prefactor before the gradient term, which makes such a computation to be quite a non-trivial problem. Below we consider the solution of this system of equations in the limit $t \rightarrow \infty$.

\section{Stationary solution of the evolution equations}

We turn now to the limit $t \rightarrow \infty$ and suppose that both the tracer velocity $V_{t r}(t)$ and the density profile around the tracer attain stationary non-zero values $V_{t r}$ and $k\left(\lambda_{1}, \lambda_{2}\right)$, i.e. $V_{t r}=$ $\lim _{t \rightarrow \infty} V_{t r}(t)$ and $k\left(\lambda_{1}, \lambda_{2}\right)=\lim _{t \rightarrow \infty} k\left(\lambda_{1}, \lambda_{2} ; t\right)$.

Next, it is expedient to rewrite Eqs(14) to (18) in terms of an auxiliary function $h_{n, m}$ :

$$
h_{n, m}=k(n \sigma, m \sigma)-\rho_{L},
$$

which determines the local deviation of the density from the average value $\rho_{L}$. In terms of this auxiliary function Eqs 14 ) to (18) become for $(n, m) \neq(0,0),( \pm 1,0),(0, \pm 1)$ 


$$
(1+\mathcal{P}) h_{n+1, m}+h_{n-1, m}+h_{n, m+1}+h_{n, m-1}-4\left(1+\frac{\mathcal{P}}{4}+\delta\right) h_{n, m}=0,
$$

while $h_{n, m}$ in the immediate vicinity of the tracer obey

$$
\begin{gathered}
(1+\mathcal{P}) h_{2,0}+h_{1,1}+h_{1,-1}-(3+4 \delta) h_{1,0}+\mathcal{P} \rho_{L}=0, \\
h_{-2,0}-(1+\mathcal{P}) h_{-1,0}+h_{-1,1}+h_{-1,-1}-(3+4 \delta) h_{-1,0}-\mathcal{P} \rho_{L}=0, \\
(1+\mathcal{P}) h_{1,1}+h_{-1,1}+h_{0,2}-(3+4 \delta) h_{0,1}=0, \\
(1+\mathcal{P}) h_{1,-1}+h_{-1,-1}+h_{0,-2}-(3+4 \delta) h_{0,-1}=0,
\end{gathered}
$$

where $D_{0}$ denotes the diffusion coefficient of an isolated adsorbed particle: $D_{0}=l \sigma^{2} / 4 \tau^{*}$, the characteristic parameter $\mathcal{P}=V_{t r} \sigma / D_{0}$ is akin to the so-called "Peclet " number in the hydrodynamics, and we have the parameter $\delta=\sigma^{2}(f+g) / 4 D_{0} \tau^{*}$. The parameter $\delta$ can be also written as $\delta=(f+g) / l=\left(1-\rho_{L}\right)^{-1}(g / l)$, where the factor $(g / l)$ is an exponential of the difference of the energy barriers against lateral diffusion and desorption. Hence, $\delta$ compares the relative weights of the adsorption/desorption and the diffusion events. Note, that the factor $(g / l)$ is usually small for most of realistic experimental situations, such that $\delta$ is small provided that $\rho_{L} \ll 1$. On the other hand, at high adsorbate densities, $\delta$ can attain relatively large values. As well, the Peclet-type number $\mathcal{P}$ can also be small or large, depending on the physical situation. Below we will consider different possible limits for $\mathcal{P}$ and $\delta$ in order to elucidate the asymptotic behavior of the tracer velocity.

In a standard approach, Eqs.(20) to (24) can be solved by introducing the generating function of the local deviation $h_{n, m}$, i.e.

$$
H(z, w)=\sum_{n=-\infty}^{\infty} \sum_{m=-\infty}^{\infty} h_{n, m} z^{n} w^{m},
$$

Multiplying Eqs.(20) to (24) by $z^{n}$ and $w^{m}$ and summing over all $n$ and $m$, we find then that the generating function $H(z, w)$ is given explicitly by

$$
H(z, w)=-K(z, w)\left\{z+\left(u-4\left(1+\frac{\mathcal{P}}{4}+\delta\right)\right)+\frac{(1+\mathcal{P})}{z}\right\}^{-1},
$$

where $u=w+1 / w$, and

$$
K(z, w)=\left((1+\mathcal{P}) h_{1,0}+\mathcal{P} \rho_{L}\right)(z-1)+h_{0,1}(u-2)+\left(h_{-1,0}-\mathcal{P} \rho_{L}\right) \frac{(1-z)}{z}
$$


Note, that $H(z, w)$ depends on $w$ only in the combination $w+1 / w$, which signifies that $h_{n, m}$ is, as it could be expected, an even function of $m$.

Now, $h_{n, m}$ can be found directly from Eqs. (26) and (27) through the standard inversion formulae [27], which amounts, however, to computating rather complex integrals. Here we will use instead a more straightforward approach, identifying $h_{n, m}$ from the expansion of $H(z, w)$ into the series in Eq.(25). To do this, we first expand $H(z, w)$ into a series in powers of $(1+\delta+\mathcal{P} / 4)^{-1}$, which gives (if $z+\frac{1+\mathcal{P}}{z}+u<4\left(1+\frac{\mathcal{P}}{4}+\delta\right)$ which is always possible)

$$
H(z, w)=K(z, w) \sum_{i=0}^{\infty}\left(4\left(1+\frac{\mathcal{P}}{4}+\delta\right)\right)^{-i-1}\left(z+\frac{(1+\mathcal{P})}{z}+u\right)^{i}
$$

Next, expanding $\left(z+(1+\mathcal{P}) z^{-1}+u\right)^{i}$ in series in powers of $w$, we rewrite Eq. 28) as a multiple series of the form

$$
H(z, w)=K(z, w) \sum_{i=0}^{\infty} \sum_{k=0}^{\infty}\left(4\left(1+\frac{\mathcal{P}}{4}+\delta\right)\right)^{-i-k-1}\left(\begin{array}{l}
i+k \\
k
\end{array}\right)\left(z+(1+\mathcal{P}) \frac{1}{z}\right)^{i} \sum_{j=0}^{k}\left(\begin{array}{l}
k \\
j
\end{array}\right) w^{k-2 j}
$$

where $\left(\begin{array}{c}i \\ k\end{array}\right)$ denote the binomial coefficients. Lastly, gathering the terms with equal powers of $z$ and $w$, we find from Eq. 29) that $h_{n, m}$ is given explicitly by

$$
h_{n, m}=\frac{\left[-(1+\mathcal{P}) h_{1,0} \nabla_{n} F_{n-1, m}+h_{-1,0} \nabla_{n} F_{n, m}+h_{0,1} \triangle_{m} F_{n, m}-\mathcal{P} \rho_{L}\left(F_{n+1, m}-F_{n-1, m}\right)\right]}{4(1+\delta+\mathcal{P} / 4)},
$$

where

$$
F_{n, m}=(1+\mathcal{P})^{-n / 2} P(n, m \mid \zeta)
$$

with

$$
\begin{aligned}
P(n, m \mid \zeta) & =\int_{0}^{\infty} d t e^{-t} I_{n}\left(\frac{\sqrt{1+\mathcal{P}} t}{2(1+\delta+\mathcal{P} / 4)}\right) I_{m}\left(\frac{t}{2(1+\delta+\mathcal{P} / 4)}\right)= \\
& =\frac{1}{(2 \pi)^{2}} \int_{-\pi}^{\pi} \int_{-\pi}^{\pi} d^{2} \mathbf{k} \frac{e^{\left(-i\left(k_{x} n+k_{y} m\right)\right)}}{1-\zeta \lambda(\mathbf{k})},
\end{aligned}
$$

in which equation $I_{n}(x)$ denotes the modified Bessel function, while the parameter $\zeta$ (which appears to be an important control parameter and which will be repeatedly used in what follows) is defined as

$$
\zeta=\frac{1+\sqrt{1+\mathcal{P}}}{2(1+\delta+\mathcal{P} / 4)}
$$

and

$$
\lambda(\mathbf{k})=\frac{\cos \left(k_{y}\right)+\sqrt{1+\mathcal{P}} \cos \left(k_{x}\right)}{1+\sqrt{1+\mathcal{P}}}
$$


Note, that $P(n, m \mid \zeta)$ can be thought off, in view of the form of Eq.(32), as the generating function for the probability of occupancy of the site $(n, m)$ (the lattice Green function) for a particular type of two-dimensional Pólya random walk, whose structure function is defined by Eq. 34) (see, e.g. Refs.[28 and [29] for more details).

Now, two comments on the just-derived expression for $h_{n, m}$, Eq.(30), are in order. First of all, we note that Eq. (30) is not closed, since it contains on the right-hand-side unknown functions; namely, $h_{ \pm 1,0}$ and $h_{0,1}$. Second, all $h_{n, m}$ are functions of the tracer terminal velocity, which still remains undefined. Consequently, in the remainder we have to find $h_{ \pm 1,0}\left(V_{t r}\right)$ and $h_{0,1}\left(V_{t r}\right)$ as functions of the microscopic parameters, (such as $f, g, \tau$ and etc.), and $V_{t r}$, and then, inserting the obtained expression for $h_{1,0}\left(V_{t r}\right)$ to Eq.(3), derive a closed with respect to $V_{t r}$ equation.

As a matter of fact, the deviations from $\rho_{L}$ in the immediate vicinity of the tracer, i.e. $h_{ \pm 1,0}\left(V_{t r}\right)$ and $h_{0,1}\left(V_{t r}\right)$, can be found directly from Eq.(30). Setting $n= \pm 1, m=0$ and $n=0, m=1$, we find from Eq.(30) that $h_{ \pm 1,0}\left(V_{t r}\right)$ and $h_{0,1}\left(V_{t r}\right)$ obey the following system of linear algebraic equations

$$
\tilde{A}\left(V_{t r}\right) \times \tilde{h}\left(V_{t r}\right)=\mathcal{P} \rho_{L} \tilde{F}\left(V_{t r}\right)
$$

where the column-matrices $\tilde{h}\left(V_{t r}\right)$ and $\tilde{F}\left(V_{t r}\right)$ are defined as

$$
\tilde{h}\left(V_{t r}\right)=\left(\begin{array}{c}
h_{1,0} \\
h_{-1,0} \\
h_{0,1}
\end{array}\right) \quad \tilde{F}\left(V_{t r}\right)=\left(\begin{array}{c}
F_{2,0}-F_{0,0} \\
F_{0,0}-F_{-2,0} \\
F_{1,1}-F_{-1,1}
\end{array}\right)
$$

while the square matrix $\tilde{A}\left(V_{t r}\right)$ has the form

$$
\tilde{A}\left(V_{t r}\right)=\left(\begin{array}{ccc}
-(1+\mathcal{P})\left(\alpha_{\zeta}+\nabla_{n} F_{0,0}\right) & \nabla_{n} F_{1,0} & \triangle_{m} F_{1,0} \\
-(1+\mathcal{P}) \nabla_{n} F_{-2,0} & -\alpha_{\zeta}+\nabla_{n} F_{-1,0} & \triangle_{m} F_{-1,0} \\
-(1+\mathcal{P}) \nabla_{n} F_{-1,1} & \nabla_{n} F_{0,1} & -\alpha_{\zeta}+\triangle_{m} F_{0,1}
\end{array}\right)
$$

where $\alpha_{\zeta}=2(1+\sqrt{1+\mathcal{P}}) / \zeta$ and $\nabla_{n}\left(\triangle_{m}\right)$ are the finite difference operators of unit step in Eq.(7) ((11)), acting on the variable $n(m)$. To define the tracer terminal velocity, we merely have to know $h_{1,0}\left(V_{t r}\right)$. It follows from Eqs. (35) to (37) that $h_{1,0}\left(V_{t r}\right)$ is given by

$$
h_{1,0}\left(V_{t r}\right)=\mathcal{P} \rho_{L} \frac{\operatorname{det} \tilde{\mathrm{h}}_{1,0}\left(\mathrm{~V}_{\mathrm{tr}}\right)}{\operatorname{det} \tilde{\mathrm{A}}\left(\mathrm{V}_{\mathrm{tr}}\right)}
$$

where $\tilde{h}_{1,0}\left(V_{t r}\right)$ is the matrix obtained from (37) by replacing the first column by $\tilde{F}\left(V_{t r}\right)$.

Now, we are in position to obtain a closed equation, which determines implicitly the tracer particle terminal velocity in terms of the parameters $f, g, \rho_{L}$ and $D_{0}$. Substituting Eqs.(38) and (19) into Eq.(3), we find that $V_{t r}$ obeys

$$
V_{t r}=\frac{\sigma\left(1-\rho_{L}\right)}{\tau}\left[1+\frac{\sigma^{2} \rho_{L}}{D_{0} \tau} \frac{\operatorname{det} \tilde{\mathrm{h}}_{1,0}\left(\mathrm{~V}_{\mathrm{tr}}\right)}{\operatorname{det} \tilde{\mathrm{A}}\left(\mathrm{V}_{\mathrm{tr}}\right)}\right]^{-1},
$$


where the first term is the mean field result for the tracer velocity (see Eq. (5)), obtained under assumption that the monolayer is perfectly stirred; terms in brackets stem from non-linear cooperative effects associated with formation of inhomogeneous density profiles, whose characteristic properties depend parametrically on the tracer velocity. Equation (39), which is the main general analytical result of our paper, is certainly too complicated to be solvable in the general case. Below we will study some of its asymptotical solutions.

\section{$5 \quad$ Asymptotic behavior of the tracer stationary velocity and density profiles at a fixed distance from the tracer}

In this section we consider the asymptotical behavior of the tracer particle velocity and density profiles at a fixed distance from the tracer in the limit of slow and fast particles diffusion ( $n$ and $m$ are taken arbitrary but fixed, while $D_{0}$ is allowed to vary). As well, we will also specify different regimes corresponding to the cases with $\mathcal{P} \gg 1$ and $\mathcal{P} \ll 1$, which may be realized at different monolayer densities. We note that such an analysis turns out to be rather non-trivial, which happens not only because of the non-linearity of the equations involved, but chiefly due to the non-uniformity of the asymptotical expansions with respect to the parameter $\delta$. As a matter of fact, such a non-uniformity is just the consequence of the qualitatively different behavior occurring in the system with explicitly conserved $(\delta=0)$ and non-conserved $(\delta>0)$ particles number. Consequently, the results for the system without adsorption and desorption can not be obtained as a limit $\delta \rightarrow 0$ of the corresponding results with arbitrary (even vanishingly small) values of $\delta$. Lastly, we note that the limits $D_{0} \rightarrow \infty$ (or $D_{0} \rightarrow 0$ ) and $n, m \rightarrow \infty$ do not commute; hence, we consider the asymptotical behavior of the density profiles at large distances from the tracer separately in Section 6 .

\subsection{Limit of a small particles diffusivity.}

Consider first the asymptotical behavior of $V_{t r}$ in the limit of a vanishingly small diffusion coefficient $D_{0}$ of the monolayer particles, $D_{0} \rightarrow 0$, which situation can be thought off as a weakly perturbed original Langmuir model $\left(D_{0}=0\right)$. It appears, as we have already remarked, that this asymptotical behavior can be different in the case when the particles number in the monolayer is not explicitly conserved, i.e. when both $f, g>0$, and in the case with a conserved particles

number, when both $f, g=0$ but their ratio is fixed, $f / g=\rho_{L} /\left(1-\rho_{L}\right)$. Consequently, we have to study these two cases separately.

A. Non-conserved particles number. We assume first that both $f$ and $g$ are non-zero, such that $\delta \neq 0$, which means that adsorption and desorption do change the occupation of each site. In this case, it seems natural to expect that the tracer will never be blocked and will always continue moving at a constant velocity. In other words, we anticipate that in this case the velocity attains a constant non-zero value $V^{(0)}>0$ as $D_{0} \rightarrow 0$ and hence, that $\mathcal{P} \gg 1, \delta \gg 1$, which means that $\zeta \ll 1$. We will refer to this limit of $D_{0} \rightarrow 0, \delta \gg 1$ and $\mathcal{P} \gg 1$ as the limit (a). We seek then $V_{t r}$ in the form

$$
V_{t r}=V^{(0)}+V^{(1)}\left(\frac{D_{0} \tau^{*}}{\sigma^{2}}\right)+\mathcal{O}\left(\left(\frac{D_{0} \tau^{*}}{\sigma^{2}}\right)^{2}\right)
$$


Further on, expanding the denominator in Eq. (32) in powers of the parameter $\zeta$, we calculate to the first order in powers of $D_{0} \tau^{*} / \sigma^{2}$ the functions $F_{n, m}$ involved in Eq.(39). These are listed in Appendix 1. Then, substituting the obtained expressions for $F_{n, m}$ into Eq.(39), we have then

$$
V^{(0)}=\frac{\left(1-\rho_{L}\right) \sigma}{\tau}\left\{1+\frac{\rho_{L} \tau^{*}}{(f+g) \tau}\right\}^{-1}
$$

and

$$
V^{(1)}=\rho_{L}\left(1-\rho_{L}\right) \sigma \frac{\rho_{L}+2+3(f+g) \tau / \tau^{*}}{\tau^{*}\left(1+(f+g) \tau / \tau^{*}\right)\left(\rho_{L}+(f+g) \tau / \tau^{*}\right)^{2}}
$$

Further on, using Eqs.(41) and (42), we find the following explicit results for the deviations from the equilibrium mean density $\rho_{L}$ in the immediate vicinity of the tracer. These obey, in the limit (a):

$$
\begin{gathered}
h_{1,0}=\frac{\rho_{L}\left(1-\rho_{L}\right)}{\rho_{L}+(f+g) \tau / \tau^{*}}\left\{1-\frac{\tau}{\tau^{*}} \frac{\left(\rho_{L}+2+3(f+g) \tau / \tau^{*}\right)}{\left(1+(f+g) \tau / \tau^{*}\right)\left(\rho_{L}+(f+g) \tau / \tau^{*}\right)}\left(\frac{D_{0} \tau^{*}}{\sigma^{2}}\right)\right\}+\mathcal{O}\left(\left(\frac{D_{0} \tau^{*}}{\sigma^{2}}\right)^{2}\right), \\
h_{-1,0}=-\frac{\rho_{L}\left(1-\rho_{L}\right)}{1+(f+g) \tau / \tau^{*}}\left\{1-\frac{\tau}{\tau^{*}} \frac{\left(\rho_{L}+2+3(f+g) \tau / \tau^{*}\right)}{\left(1+(f+g) \tau / \tau^{*}\right)^{2}}\left(\frac{D_{0} \tau^{*}}{\sigma^{2}}\right)\right\}+\mathcal{O}\left(\left(\frac{D_{0} \tau^{*}}{\sigma^{2}}\right)^{2}\right),
\end{gathered}
$$

and

$$
h_{0,1}=\frac{\rho_{L}\left(1-\rho_{L}\right)^{2}}{(f+g)\left(1+(f+g) \tau / \tau^{*}\right)^{2}}\left(\frac{D_{0} \tau^{*}}{\sigma^{2}}\right)+\mathcal{O}\left(\left(\frac{D_{0} \tau^{*}}{\sigma^{2}}\right)^{2}\right),
$$

As it could be expected intuitively, $h_{1,0}>0$ and $h_{-1,0}<0$, which means that there is a condensed, "jammed" region in front of the tracer, and a depleted region past the tracer. On the other hand, the fact that $h_{0,1}>0$ and that $h_{ \pm 1,0}$ under certain conditions are non-monotonous functions of $\rho_{L}$ appear to be non-trivial. We will discuss the characteristic properties of these two regions at the end of this section and in the section 6 .

Note, that the result in Eq.(41) can be simply obtained from the initial system of equations (20) to (24) by assuming that the deviation $h_{n, 0}$ from the mean density is zero for $n \geq 2$, i.e. the density profile in front of the density profile is an abrupt step function. Then, it follows from Eqs.(21) and (22) that $h_{ \pm 1,0} \approx \pm \mathcal{P} \rho_{L} /(3+4 \delta)$ Plugging this approximate result into Eq.(3) and turning to the limit $D_{0} \rightarrow 0$ we actually recover Eq.(41). Correction term in Eq.(42) is thus associated with the appearance of a smooth, long-range density variation with the distance from the tracer due to diffusion, which couples effectively the evolution of $h_{n, m}$ at different lattice sites. Note finally, that Eq.(41) reduces to the essentially mean-field in Eq.(5), i.e. $V_{t r}=\sigma\left(1-\rho_{L}\right) / \tau$, in the limit $\tau^{*} \rightarrow 0$, i.e. in the limit when adsorption/desorption processes proceed at infinitely fast rate resulting in a complete homogenization of the monolayer.

Next, using Eq. (30), and taking advantage of the fact that to the lowest non-trivial order in powers of $D_{0}$ the function $F_{n, m}$ in Eq.(31) is given by 


$$
F_{n, m} \sim\left(\begin{array}{l}
|n|+|m| \\
|n|
\end{array}\right)\left(\frac{D_{0}}{V^{(0)} \sigma+\sigma^{2}(f+g) / \tau^{*}}\right)^{|n|+|m|} J_{n},
$$

where

$$
J_{n}=\left\{\begin{array}{l}
1, \text { if } n>0, \\
\left(V^{(0)} \sigma / D_{0}\right)^{|n|}, \text { if } n<0,
\end{array}\right.
$$

we find that, at a finite distance from the tracer, the local deviation from the equilibrium mean density obey:

$$
\begin{gathered}
h_{n, m} \sim \frac{\rho(1-\rho)}{\rho+(f+g) \tau / \tau^{*}}\left(\begin{array}{l}
n-1+|m| \\
n-1
\end{array}\right)\left[\frac{D_{0} \tau^{*}}{\sigma^{2}(f+g)} \frac{\rho+(f+g) \tau / \tau^{*}}{1+(f+g) \tau / \tau^{*}}\right]^{n-1+|m|} \text { for } n>0, \\
h_{n, m} \sim-\frac{\rho(1-\rho)}{1+(f+g) \tau / \tau^{*}}\left(\begin{array}{l}
|n|+|m| \\
|n|
\end{array}\right)\left[\frac{1-\rho}{1+(f+g) \tau / \tau^{*}}\right]^{|n|-1}\left[\frac{D_{0} \tau^{*}}{\sigma^{2}(f+g)} \frac{\rho+(f+g) \tau / \tau^{*}}{1+(f+g) \tau / \tau^{*}}\right]^{|m|} \text { for } n<0,
\end{gathered}
$$

and

$$
h_{0, m} \sim \frac{|m| \rho(1-\rho)^{2}}{\left(1+(f+g) \tau / \tau^{*}\right)\left(\rho+(f+g) \tau / \tau^{*}\right)}\left[\frac{D_{0} \tau^{*}}{\sigma^{2}(f+g)} \frac{\rho+(f+g) \tau / \tau^{*}}{1+(f+g) \tau / \tau^{*}}\right]^{|m|},
$$

Note, that these expressions generalize the leading order contributions of Eqs.(70)-(45). It may be also worthy to remark that $h_{n, m}$ turns out to be a non-separable function with respect to the variables $n$ and $m$. The density profiles defined by Eqs. (47) to (49) are depicted in Fig.2a.

Lastly, we comment on the range of applicability of all the results of this subsection, which are based on the assumption that $\mathcal{P} \gg 1$ and $\zeta \ll 1$. This implies, in turn, that these results are only valid when $D_{0}$ obeys the inequality

$$
D_{0} \ll \frac{\sigma^{2}\left(1-\rho_{L}\right)}{2 \tau}\left\{1+\frac{\rho_{L} \tau^{*}}{(f+g) \tau}\right\}^{-1}
$$

which sets up an upper bound on $D_{0}$ showing how small it should be to insure the utility the previous expressions.

B. Conserved particles number. Consider next the special case when both $f, g \rightarrow 0$, but their ratio $f / g$ is kept fixed, $f / g=\rho_{L} /\left(1-\rho_{L}\right)$, which corresponds to the situation with a conserved number of particles (no adsorption/desorption), i.e. a standard model of a two-dimensional hardcore lattice gas. In this case the parameter $\delta$ is exactly equal to zero, $\delta=0, V^{(0)}$ should vanish according to Eq.(41), and hence, $V_{t r}$ is expected to scale linearly with $D_{0}$ in the lowest order with respect to the particle diffusion coefficient. Note, however, that in the case $\delta=0$ the inequality in Eq.(50) is not satisfied for any $D_{0}$ except $D_{0}=0$, which means that, in principle, Eqs.(41) and (42) do not describe the behavior of the tracer velocity properly.

Let us now examine separately the case $\delta=0$ for which the results (41) to (45) do not apply. Assume first that the Peclet number is large, i.e. $\mathcal{P} \gg 1$, which will be checked for consistency afterwards. Then, in this limit $D_{0} \rightarrow 0, \delta=0$ and $\mathcal{P} \gg 1$, which will be referred to in the remainder as the limit (b), we have that $\zeta \ll 1$ and we can use the expressions for $F_{n, m}$ (see 
Appendix 1) with $\delta=0$, provided that $V^{(0)}$ is replaced by $V_{t r}$. This yields for the terminal velocity the following result

$$
V_{t r} \sim \frac{2\left(1-\rho_{L}\right)}{\sigma \rho_{L}} D_{0}
$$

It follows then from Eq.(51) that here the Peclet number is given approximately by $\mathcal{P} \approx 2(1-$ $\left.\rho_{L}\right) / \rho_{L}$; hence, Eq. (51) is consistent with the underlying assumption only when $\rho_{L} \ll 1$. In this limit, we find that $h_{1,0} \sim 1, \quad h_{-1,0} \sim-\rho_{L}, \quad$ and $\quad h_{0,1} \sim \rho_{L} / 2$. Note that in this case, $h_{0,1}$ is independent of $D_{0}$ to leading order, contrarily to the prediction of Eq. (45).

More generally, using Eq. (30) and Eqs.(95)- (100), we find that at a finite distance from the tracer

$$
\begin{gathered}
h_{n, m} \sim\left(\begin{array}{c}
n-1+|m| \\
n-1
\end{array}\right)\left(\frac{\rho}{2}\right)^{n-1+|m|} \text { for } n>0, \\
h_{n, m} \sim-\left(\begin{array}{l}
|n|+|m| \\
|n|
\end{array}\right) \frac{|m|}{|m|-n+1}\left(\frac{\rho}{2}\right)^{|m|} \text { for } n<0 \text { and } m \neq 0
\end{gathered}
$$

and

$$
h_{0, m} \sim|m|\left(\frac{\rho}{2}\right)^{|m|} .
$$

Furthermore, using the expansion

$$
F_{n, 0}=1+\frac{2(n+1)}{\mathcal{P}}+\mathcal{O}\left(\frac{1}{\mathcal{P}^{2}}\right) \text { for } \delta=0 \text { and } n<0,
$$

we have

$$
h_{n, 0} \sim-\rho \text { for } n<0
$$

Again, these expressions are not separable in $n$ and $m$. The density profiles defined by Eqs. (52) to (56) are depicted in Fig.2b.

Let us consider now the limit (c) when $D_{0} \rightarrow 0, \delta=0$, but $\mathcal{P} \ll 1$. In this limit we evidently have $\zeta \sim 1$. Notice now that for $\zeta=1$, the integral in Eq.(32) diverges at small values of the wave-vector $\mathbf{k}$, so that when $1-\zeta$ is small but non-zero, the integral over $\mathbf{k}$ is dominated by the small-k behavior (strictly speaking, this is a priori true only if $n$ and $m$ are large enough to insure the validity of the stationary phase method). Following Montroll and Weiss [28], who have studied asymptotic behavior of $P(n, m \mid \zeta)$ in Eq. (32) within the context of Pólya random walk on 2D lattices, (see also Ref.[29]), we first represent the structure function in Eq.(34) as $1-\lambda(\mathbf{k}) \sim \frac{1}{2}\left(\sigma_{x}^{2} k_{x}^{2}+\sigma_{y}^{2} k_{y}^{2}\right)$, where

$$
\sigma_{x}=\frac{(1+\mathcal{P})^{1 / 4}}{(1+\sqrt{1+\mathcal{P}})^{1 / 2}} ; \sigma_{y}=\frac{1}{(1+\sqrt{1+\mathcal{P}})^{1 / 2}}
$$

Then, using the stationary phase method, one finds [29] the following representation of $P(n, m \mid \zeta)$ in Eq.(32), which holds in the limit $\zeta \rightarrow 1$ :

$$
P(n, m \mid \zeta) \sim \frac{1}{\pi \sigma_{x} \sigma_{y}} K_{0}\left(\left[2(1-\zeta)\left(\frac{n^{2}}{\sigma_{x}^{2}}+\frac{m^{2}}{\sigma_{y}^{2}}\right)\right]^{1 / 2}\right)
$$


where $K_{0}(z)$ is the modified Bessel function of the third kind. In the limit $\zeta \rightarrow 1$, Eq. (58) yields:

$$
P(n, m \mid \zeta)=-\frac{2}{\pi} \ln \left(\frac{\mathcal{P}}{4}\right)-\frac{\ln \left(n^{2}+m^{2}\right)}{\pi}-\frac{2 \gamma}{\pi}+\mathcal{O}(\mathcal{P} \ln \mathcal{P})
$$

Note that Eq. (59) only holds at intermediate distances from the origine, since, on the one hand, $n$ and $m$ have to be large enough in order to apply th stationary phase method, but, on the other hand, the argument of $K_{0}$ in Eq. (58) should be small. However, as shown in Ref. 30], the asymptotical result in Eq.(59) serves as a very good estimate when $n$ and $m$ are sufficiently large (in practical, as soon as $\left(n^{2}+m^{2}\right)^{1 / 2} \geq 3$ ). Since we are also interested to know $h_{ \pm 1,0}$ and $h_{0, \pm 1}$ precisely, we list below some particular values of $P(n, m \mid \zeta)$ in the immediate vicinity of the tracer. Exact values of $P(n, m \mid \zeta \rightarrow 1)$ are presented in Ref. 30]. From these, we find

$\nabla_{n} F_{0,0}=-1+\mathcal{O}(\mathcal{P} \ln (\mathcal{P})), \quad \nabla_{n} F_{1,0}=-3+8 / \pi+\mathcal{O}(\mathcal{P} \ln (\mathcal{P})), \quad \triangle_{m} F_{1,0}=2-8 / \pi+\mathcal{O}(\mathcal{P} \ln (\mathcal{P}))$

$\nabla_{n} F_{-2,0}=3-8 / \pi+\mathcal{O}(\mathcal{P} \ln (\mathcal{P})), \quad \nabla_{n} F_{-1,0}=1+\mathcal{O}(\mathcal{P} \ln (\mathcal{P})), \quad \triangle_{m} F_{-1,0}=2-8 / \pi+\mathcal{O}(\mathcal{P} \ln (\mathcal{P}))$

$\nabla_{n} F_{-1,1}=-1+4 / \pi+\mathcal{O}(\mathcal{P} \ln (\mathcal{P})), \quad \nabla_{n} F_{0,1}=1-4 / \pi+\mathcal{O}(\mathcal{P} \ln (\mathcal{P})), \quad \triangle_{m} F_{0,1}=-2+8 / \pi+\mathcal{O}(\mathcal{P} \ln (\mathcal{P}))$,

which allow us to determine the local deviations in the immediate vicinity of the tracer. We find that in the limit (c) the local deviations are to the leading order in $\mathcal{P}$ :

$$
h_{1,0}=\alpha \rho_{L} \mathcal{P}+\mathcal{O}\left(\mathcal{P}^{2}\right) \quad h_{-1,0}=-\alpha \rho_{L} \mathcal{P}+\mathcal{O}\left(\mathcal{P}^{2}\right) \quad h_{0,1}=\mathcal{O}\left(\mathcal{P}^{2}\right)
$$

where $\alpha$ is a numerical constant,

$$
\alpha=-\frac{P(2,0 \mid 1)-P(0,0 \mid 1)}{4+P(2,0 \mid 1)-P(0,0 \mid 1)}=\frac{\pi-2}{2} \approx 0.571
$$

Finally, inserting Eqs. 63) to Eq.(3), we find the following expression for the tracer terminal velocity in the limit $(\mathrm{c})$ :

$$
V_{t r}=\frac{\left(1-\rho_{L}\right) D_{0}}{\alpha \sigma \rho_{L}}\left[1-\frac{D_{0} \tau}{\alpha \sigma^{2} \rho_{L}}+\mathcal{O}\left(\left(\frac{D_{0} \tau^{*}}{\sigma^{2}}\right)^{2}\right)\right]
$$

Note, that similarly to Eq.(51), in the limit (c) the tracer velocity $V_{t r}$ vanishes in proportion to $D_{0}$; the prefactor is, however, different from that in Eqs.(42) and Eq.(51). The Peclet number in this case is again $\mathcal{P} \sim\left(1-\rho_{L}\right) / \rho_{L}$, which means, in view of the consistency with the initial assumption, that the limit (c) is only realized and Eq.(65) is only valid when $\rho_{L} \sim 1$. We also remark, that a similar to Eq. (65) expression for the tracer velocity with, however, a bit different value of $\alpha$, has been obtained prior in [20], which studied the stationary velocity of a driven tracer particle in a two-dimensional lattice gas with a conserved particles number. 
Lastly, using Eq. (30) and Eq. (59), we find that at a finite, but large enough distance from the tracer,

$$
h_{n, m} \sim \frac{(1-\rho)(1+\alpha)}{4 \pi \alpha} \ln \left(\frac{(n+1)^{2}+m^{2}}{(n-1)^{2}+m^{2}}\right),
$$

which is again a non-separable function of variables $n$ and $m$. Profiles defined by Eq. (66) are depicted in Fig.2c.

\subsection{Limit of a large particles diffusivity.}

We turn next to the analysis of the asymptotical behavior of $V_{t r}$ in the limit of very fast monolayer particles diffusion supposing that both $f$ and $g$ are non-zero, which will be called the limit (d). Clearly, when $D_{0}=\infty$, one should recover for $V_{t r}$ the trivial mean-field result in Eq.(5). We search, hence, for the first correction to this result, representing the tracer velocity as

$$
V_{t r}=\frac{\sigma\left(1-\rho_{L}\right)}{\tau}+V_{(1)}\left(\frac{\sigma^{2}}{D_{0} \tau}\right)+\mathcal{O}\left(\left(\frac{\sigma^{2}}{D_{0} \tau}\right)^{2}\right)
$$

Now, one can readily notice that in this case we deal, in essence, with a situation which is quite similar to the limit (c) studied in the previous subsection. Namely, both $\delta$ and $\mathcal{P}$ are small and hence, $\zeta$ should be of order of unity, $\zeta \sim 1$. Consequently, all the analysis of the last subsection applies here, except that $\zeta$ takes a bit different form.

Noticing next that here $\zeta \approx 1-\delta$ and calculating the integral in Eq.(32) along essentially the same lines as it was done in the previous subsection, we find that in the limit of fast particles diffusion $P(n, m \mid \zeta \rightarrow 1)$ is given by :

$$
P(n, m \mid \zeta \rightarrow 1) \sim-\frac{2}{\pi} \ln (\delta)-\frac{\ln \left(n^{2}+m^{2}\right)}{\pi}-\frac{2 \gamma}{\pi}+\mathcal{O}\left(\frac{\ln \left(D_{0}\right)}{D_{0}^{2}}\right),
$$

which expansion holds for large enough values of the variables $n$ and $m$. Consequently, we find that in the limit (d) the local deviations from the equilibrium mean density in the immediate vicinity of the tracer obey Eqs.(63) and hence, the first correction to Eq.(可) reads

$$
V_{(1)}=-\rho_{L}\left(1-\rho_{L}\right) \frac{\alpha \sigma}{\tau}
$$

As it could be expected intuitively, $V_{(1)}$ appears to be negative, i.e. the actual tracer terminal velocity is lower than that given by Eq.(5). Note also that $V_{(1)}$ is a non-monotonous function of $\rho_{L}$ and is maximal when $\rho_{L}=1 / 2$. We finally remind that the expansions in Eq.(67) and Eq.(69) hold, by definition, in the limit $D_{0} \rightarrow \infty$, which means they only apply when both $\mathcal{P}$ and $\delta$ are much less than unity.

We remark that Eq.(69) and Eqs.(63) still hold when $f, g=0$. On the other hand, it will be shown in the Appendix that in the limit (d) the amplitudes and the characteristic lengths of the density profiles attain different forms depending whether $\delta$ is equal to zero or not.

Lastly, the form of the density profiles at a finite distance from the tracer is very similar to the one found in the limit (c). We have that, at finite but large enough distance, $h_{n, m}$ is given by:

$$
h_{n, m} \sim \frac{\rho(1-\rho)(1+\alpha)}{4 \pi} \frac{\sigma^{2}}{\tau D_{0}} \ln \left(\frac{(n+1)^{2}+m^{2}}{(n-1)^{2}+m^{2}}\right),
$$

The remarks following Eq.(66) still hold. 


\section{Asymptotic forms of the density profiles at large dis- tances from the tracer.}

In this section we consider the asymptotical behavior of the density profiles at large distances from the stationary moving tracer particle. The first subsection deals with the simple special case $D_{0}=0$. The second subsection will be devoted to the analysis of the asymptotic forms of $h_{n, 0}$ in the limit $n \rightarrow \infty$, which will be based on our Eqs.(30) to (32). Further on, in the next subsection we will examine a more complicated question of the decay of $h_{n, 0}$ at large separations past the tracer, where we again discuss separately the behavior in the cases with non-conserved and conserved particles numbers. Actually, we observe here a very spectacular effect of a qualitative change of the asymptotical behavior; the density relaxation to the average value $\rho_{L}$ proceeds in the former case exponentially, while in the latter case it is described by a slow algebraic function of the distance. Lastly, we will study the forms of some integral characteristic of the density profile, such as, for instance, the global deviation of the density from the equilibrium value in the domains $n>1$ and $n<-1$.

Asymptotic behavior of $h_{n, 0}$ as $n \rightarrow \pm \infty$ can be most conveniently studied if we introduce the generating function of $h_{n, 0}$ of the form

$$
h(z)=\sum_{-\infty}^{+\infty} h_{n, 0} z^{n}
$$

Setting then $m=0$ in Eq.(30) and summing over all $n$, we find that $h(z)$ is given explicitly by

$$
h(z)=\frac{z(z-1)\left((1+\mathcal{P}) h_{1,0}+\mathcal{P} \rho_{L}\right)+(1-z)\left(h_{-1,0}-\mathcal{P} \rho_{L}\right)}{\sqrt{\left(z-z_{1}\right)\left(z-z_{2}\right)\left(z-z_{3}\right)\left(z-z_{4}\right)}}-h_{0,1} \sqrt{\frac{\left(z-z_{2}\right)\left(z-z_{3}\right)}{\left(z-z_{1}\right)\left(z-z_{4}\right)}}
$$

where the roots $z_{i}$ are as follows:

$$
\begin{aligned}
& z_{1}=\frac{1+\sqrt{1+\mathcal{P}}+\zeta}{\zeta}-\sqrt{\left(\frac{1+\sqrt{1+\mathcal{P}}+\zeta}{\zeta}\right)^{2}-(1+\mathcal{P})} \\
& z_{2}=\frac{1+\sqrt{1+\mathcal{P}}-\zeta}{\zeta}-\sqrt{\left(\frac{1+\sqrt{1+\mathcal{P}}-\zeta}{\zeta}\right)^{2}-(1+\mathcal{P})} \\
& z_{3}=\frac{1+\sqrt{1+\mathcal{P}}-\zeta}{\zeta}+\sqrt{\left(\frac{1+\sqrt{1+\mathcal{P}}-\zeta}{\zeta}\right)^{2}-(1+\mathcal{P})} \\
& z_{4}=\frac{1+\sqrt{1+\mathcal{P}}+\zeta}{\zeta}+\sqrt{\left(\frac{1+\sqrt{1+\mathcal{P}}+\zeta}{\zeta}\right)^{2}-(1+\mathcal{P})}
\end{aligned}
$$

and obey

$$
0<z_{1} \leq z_{2} \leq 1<z_{3} \leq z_{4},
$$

such that $h(z)$ is analytic in the annular region of inner radius $z_{2}$ and outer radius $z_{3}$. This implies that both $z_{1}$ and $z_{4}$ are irrelevant to the large scale behavior and the dominant contribution to the asymptotic form of $h_{n, 0}$ as $n \rightarrow \pm \infty$ comes from the the behavior of $h(z)$ as $z \rightarrow z_{2}$ or $z \rightarrow z_{3}$. 
More precisely, the asymptotic form $h_{n, 0}$ as $n \rightarrow+\infty$ stems from the behavior of $h(z)$ in the vicinity of $z=z_{3}$, while $h_{n, 0}$ as $n \rightarrow-\infty$ is associated with the corresponding behavior near $z=z_{2}$.

\subsection{Density profiles in the special case $D_{0}=0$.}

First of all, we study the special case $D_{0}=0$, for which the roots $z_{1}$ and $z_{2}$ collapse to the same value $\left(1-\rho_{L}\right) /\left(1+(f+g) \tau / \tau^{*}\right)$. In this special case, which corresponds to the original Langmuir model, it is possible to determine the density profile exactly. Indeed, here Eq. (72) reduces to

$$
\begin{aligned}
h(z) & =h_{1,0} \frac{(z-1)\left(z+h_{-1,0} / h_{1,0}\right)}{z\left(1-z_{2} / z\right)} \\
& =h_{1,0}\left(z-\left(1-h_{-1,0} / h_{1,0}\right)+h_{-1,0} / h_{1,0} \frac{1}{z}\right) \sum_{n=0}^{\infty}\left(\frac{z_{2}}{z}\right)^{n} \\
& =h_{1,0}\left(z+\sum_{n=1}^{\infty} \frac{\left(1-z_{2}\right) z_{2}^{n-1}}{z^{n}}\right),
\end{aligned}
$$

which implies

$$
h_{n, 0}=0, \text { for } n>1, h_{n, 0}=h_{-1,0}\left(\frac{1+(f+g) \tau / \tau^{*}}{1-\rho_{L}}\right)^{n}, \text { for } n<0,
$$

where $h_{1,0}$ and $h_{-1,0}$ are given to the leading order by Eqs.(70) and (44) respectively. These expressions confirm the step shaped profile in front of the tracer particle (as anticipated in section 5.1), such that the only perturbed sites are the sites visited by the tracer particle, and the site just in front of it. These expressions can be also readily recovered by considering the limiting form of the equations (20)- (24) when $D_{0}=0$.

\subsection{Asymptotic forms of the density profiles at large distances in front of the stationary moving tracer.}

From now on, we assume that the diffusion coefficient $D_{0}$ is not equal to zero, and we deduce the asymptotical behavior of $h_{n, 0}$ in the limit $n \rightarrow \pm \infty$ from the analysis of the singularities of the generating function $h(z)$. One has that in the vicinity of $z=z_{3}$ the generating function $h(z)$ obeys

$$
h(z)=\frac{z_{3}\left(z_{3}-1\right)\left((1+\mathcal{P}) h_{1,0}+\mathcal{P} \rho_{L}\right)+\left(1-z_{3}\right)\left(h_{-1,0}-\mathcal{P} \rho_{L}\right)}{\sqrt{\left(z_{3}-z_{1}\right)\left(z_{3}-z_{2}\right)\left(z_{4}-z_{3}\right)}} \frac{1}{\sqrt{z_{3}-z}}+\mathcal{O}\left(\sqrt{z_{3}-z}\right)
$$

Next, following Darboux method [27] or singularity analysis of generating functions [31, we find that $h_{n, 0}$ in the limit $n \rightarrow \infty$ follows

$$
h_{n, 0} \sim \frac{z_{3}\left(z_{3}-1\right)\left((1+\mathcal{P}) h_{1,0}+\mathcal{P} \rho_{L}\right)+\left(1-z_{3}\right)\left(h_{-1,0}-\mathcal{P} \rho_{L}\right)}{\sqrt{z_{3}\left(z_{3}-z_{2}\right)\left(z_{4}-z_{3}\right)\left(z_{3}-z_{1}\right)}} \frac{1}{\sqrt{\pi n}} \frac{1}{z_{3}^{n}}
$$

For notational convenience, the latter expression can be rewritten as 


$$
h_{n, 0} \sim K_{+} \frac{\exp \left(-n / \lambda_{+}\right)}{n^{1 / 2}}
$$

where the decay amplitude is given by

$K_{+}=\frac{\left(z_{3}-1\right)\left((1+\mathcal{P}) h_{1,0}+\mathcal{P} \rho_{L}\right)+\left(1 / z_{3}-1\right)\left(h_{-1,0}-\mathcal{P} \rho_{L}\right)}{\sqrt{8 \pi}}\left[\left(\frac{1+\sqrt{1+\mathcal{P}}-\zeta}{\zeta}\right)^{2}-(1+\mathcal{P})\right]^{-1 / 4}$,

while the characteristic decay length obeys

$$
\lambda_{+}=1 / \ln \left(z_{3}\right)
$$

Therefore, the density in front of the stationary moving tracer approaches the equilibrium value $\rho_{L}$ exponentially with the distance.

Note now that the decay amplitude $K_{+}$and the characteristic length $\lambda_{+}$, Eqs. (80) and (81), depend on $\zeta$ and on $h_{ \pm 1,0}$. It may be thus instructive to analyze the asymptotical forms of $K_{+}$and $\lambda_{+}$, using the explicit results for $h_{ \pm 1,0}$ obtained in the previous section. This analysis is presented in Appendix 2.

\subsection{Asymptotic forms of the density profiles at large distances past the stationary moving tracer.}

We first note that one of the roots of the generating function, namely $z_{2}$, gets equal to unity when both $f$ and $g$ are strictly equal to zero, which results in the exact cancellation of the multiplier $1-z_{3}$ both in the nominator and the denominator in Eq.(71). This shows that in the limit when exchanges with the reservoir are forbidden, qualitative changes in the singular behavior of the generating function at the vicinity of $z_{2}$ will appear as compared to the case when $f, g>0$. Consequently, we have to consider separately the behavior in the case of non-conserved particles number, when exchanges with the reservoir persist, and the case when both $f$ and $g$ are equal to zero while their ratio is kept fixed.

A. Non-conserved particles number. From Eq.(71) one finds that in the vicinity of $z=z_{2}$ the generating function $h(z)$ behaves as

$$
h(z)=\frac{z_{2}\left(z_{2}-1\right)\left((1+\mathcal{P}) h_{1,0}+\mathcal{P} \rho_{L}\right)+\left(1-z_{2}\right)\left(h_{-1,0}-\mathcal{P} \rho_{L}\right)}{\sqrt{\left(z_{2}-z_{1}\right)\left(z_{3}-z_{2}\right)\left(z_{4}-z_{2}\right)}} \frac{1}{\sqrt{z-z_{2}}}+\mathcal{O}\left(\sqrt{z-z_{2}}\right),
$$

which implies the following form of $h_{n, 0}$ in the limit $n \rightarrow-\infty$,

$$
h_{n, 0} \sim K_{-} \frac{\exp \left(n / \lambda_{-}\right)}{(-n)^{-1 / 2}}
$$

with

$K_{-}=\frac{\left(z_{2}-1\right)\left((1+\mathcal{P}) h_{1,0}+\mathcal{P} \rho_{L}\right)+\left(1 / z_{2}-1\right)\left(h_{-1,0}-\mathcal{P} \rho_{L}\right)}{\sqrt{8 \pi}}\left[\left(\frac{1+\sqrt{1+\mathcal{P}}-\zeta}{\zeta}\right)^{2}-(1+\mathcal{P})\right]^{-1 / 4}$, 
and

$$
\lambda_{-}=-1 / \ln \left(z_{2}\right)
$$

B. Conserved particles number. Suppose now that both $f$ and $g$ are equal to zero, i.e. $\delta=$ 0 , while their ratio is fixed and given by $f / g=\rho_{L} /\left(1-\rho_{L}\right)$. As we have already remarked, this situation corresponds to the usual model of a two-dimensional hard-core lattice gas without exchanges with a reservoir. Here, the generating function in the vicinity of its singular point $z=z_{2}=1$ obeys

$$
h(z)=\left[\frac{2 \mathcal{P} \rho_{L}+(1+\mathcal{P}) h_{1,0}-h_{-1,0}}{\sqrt{z_{3}-1}}-h_{0,1} \sqrt{z_{3}-1}\right] \frac{1}{\sqrt{\left(1-z_{1}\right)\left(z_{4}-1\right)}} \sqrt{z-1}+\mathcal{O}\left((z-1)^{3} / 2\right),
$$

which yields the following result for $h_{n, 0}$ in the limit $n \rightarrow-\infty$,

$$
h_{n, 0} \sim-\frac{K_{-}}{n^{3 / 2}}\left(1+\frac{3}{8 n}+\mathcal{O}\left(\frac{1}{n^{2}}\right)\right),
$$

where

$$
K_{-}=\frac{1}{4 \sqrt{\pi}}\left(\frac{2 \mathcal{P} \rho_{L}+(1+\mathcal{P}) h_{1,0}-h_{-1,0}}{\sqrt{\mathcal{P}}}-h_{0,1} \sqrt{\mathcal{P}}\right)
$$

Remarkably enough, in this case the correlations between the tracer position and the particles distribution vanish only algebraically slow with the distance! This implies, in turn, that in the conserved particles number case, the mixing of the monolayer is not efficient and the medium "remembers" the passage of the tracer for a long time which signifies strong memory effects. We note also that the algebraic decay of correlations in this model has been predicted earlier in Ref. 20]. However, the decay exponent has been erroneously predicted to be equal to $1 / 2$, as opposed to the value $3 / 2$ given by Eq. 87). As well, the amplitude $K_{-}$happens to have a different sign, compared to that in Ref.[20], which invalidates the conclusion that the overall relaxation to the equilibrium value $\rho_{L}$ is a non-monotonic function of the distance.

Asymptotic forms of $K_{-}$and $\lambda_{-}$in different limiting cases are presented in Appendix 2.

\subsection{Integral characteristics of the density profiles}

First of all, we address the question whether the driven tracer, which induces an inhomogeneous density distribution in the monolayer, shifts the equilibrium between adsorption and desorption, i.e. whether it changes effectively the equilibrium density in the monolayer. The answer is trivially "no" in the case when the particles number is explicitly conserved, but in the general case with arbitrary $f$ and $g$ this is not at all evident: desorption events are certainly favored in front of the tracer, while the adsorption events are evidently suppressed by the excess density. On the other hand, past the tracer desorption is diminished due to the particles depletion while adsorption may proceed more readily due to the same reason. It is thus not at all clear a priori whether these two effects can compensate each other exactly, in view of the asymmetry of the density profiles.

For this purpose, we study first the behavior of the integral deviation $\Omega$ of the density from the equilibrium value $\rho_{L}$, i.e.

$$
\Omega=\sum_{n=-\infty}^{+\infty} \sum_{m=-\infty}^{+\infty} h_{n, m}
$$


As a matter of fact, this integral characteristic can be computed straightforwardly from Eq.26. Setting $z$ and $w$ equal to unity, we find that even in the case when $\delta \neq 0$, the integral deviation $\Omega=0$. This means that the inhomogeneity of the density distribution in the monolayer created by the tracer does not perturb the global balance between the adsorption and desorption events. An analogous result has been obtained for the one dimensional problem in Ref. 21.

Next we would like to check whether such a compensation holds on the axis of the tracer motion, i.e. whether the integral deviation $\Omega_{X}$ along the $X$-axis is zero or not. Setting $z=1$ in Eq.(71), we find then the following general result

$$
\Omega_{X}=\sum_{n=-\infty}^{\infty} h_{n, 0}=-\sqrt{\frac{\delta}{1+\delta}} h_{0,1}
$$

which implies that such an exact compensation is not realized on the $X$-axis only, except for the case $\delta=0$, which also does not seem to be a trivial fact. Moreover, it follows from Eq. (90) that $\Omega_{X}$ is always negative, provided that $h_{0,1}$ is positive definite, which means apparently that on the $X$-axis the equilibrium is shifted towards desorption. Note, however, that $\Omega_{X}$ always remains small and tends to zero in the limits $D_{0} \rightarrow 0$ and $D_{0} \rightarrow \infty$; hence, $\Omega_{X}$ should be a bell-shaped function of the particles diffusivity.

Finally, we look at the total excess "weight" of the condensed region in front of the tracer particle, defined as

$$
\Omega_{X+}=\sum_{n=1}^{\infty} h_{n, 0}
$$

Using the expression for $h_{n, m}$ in Eq.(30), we find for $\Omega_{X+}$ the following asymptotical results:

(a) In the limit of small particles diffusivity and $\delta \neq 0(\mathcal{P} \gg 1)$,

$$
\Omega_{X+} \sim \frac{\rho_{L}\left(1-\rho_{L}\right)}{\rho_{L}+(f+g) \tau / \tau^{*}} .
$$

This quantity is smaller than one, but finite in the general case. Moreover, it appears to be nonmonotonic with respect to $\rho_{L}$. Lastly, it tends to zero if $\tau^{*}$ tends to zero, i.e. when the exchanges with the vapour phase proceed infinitely fast.

(b) In the limit of small particles diffusivity, $\delta=0$ and $\mathcal{P} \ll 1, \Omega_{X+} \sim 1$, which result can be obtained from the previous one 92 by taking the limit $\delta \rightarrow 0$, and recollecting that $\rho_{L} \ll 1$ in the limit (b).

(c)In the limit of small particles diffusivity, $\delta=0$ and $\mathcal{P} \gg 1$,

$$
\Omega_{X+} \sim \frac{(1+\alpha)\left(1-\rho_{L}\right)}{\alpha \pi} \ln \left(\frac{1}{1-\rho_{L}}\right) .
$$

Since here $\rho_{L} \sim 1, \Omega_{X+}$ appears to be small.

(d) In the limit of fast particles diffusion $(\mathcal{P} \gg 1), \Omega_{X}$ approaches a constant value

$$
\Omega_{X+} \sim \frac{(1+\alpha) \sigma^{2} \rho_{L}\left(1-\rho_{L}\right)}{\pi D_{0} \tau} \ln \left(\frac{D_{0} \tau}{\sigma^{2}\left(1-\rho_{L}\right)}\right)
$$

Note that $\Omega_{X+}$ is non-monotonic with respect to $\rho_{L}$, which generalizes the result announced in [20] to the case with non-conserved particles number. Note also that $\Omega_{X+} \rightarrow 0$ as $D_{0} \rightarrow \infty$, which shows that the condensed region disappears if the diffusion processes become efficient enough to mix the adsorbed monolayer. 


\section{Conclusions}

To conclude, we have studied analytically dynamics of a driven probe molecule in a two-dimensional adsorbed monolayer composed of mobile, hard-core particles undergoing continuous exchanges with the vapour phase. Our analytical approach was based on the master equation, describing the time evolution of the system, which allowed us to evaluate a system of coupled dynamical equations for the tracer particle velocity and a hierarchy of correlation functions. To solve these coupled equations, we have invoked an approximate closure scheme based on the decomposition of the third-order correlation functions into a product of pairwise correlations, which has been first introduced in Ref. 23] for a related model of driven tracer dynamics in a one-dimensional lattice gas with conserved particles number. Within the framework of this approximation, we have derived a system of coupled, discrete-space equations describing evolution of the density profiles, as seen from the moving probe, and its velocity $V_{t r}$. We have shown then that $V_{t r}$ depends on the monolayer particles density in front of the tracer, which is itself dependent on the magnitude of the velocity, as well as on the rate of the adsorption/desorption processes and the rate at which the particles can diffuse away of the tracer. As a consequence of such a non-linear coupling, in the general case, (i.e. for arbitrary adsorption/desorption rates and particles diffusion coefficient), $V_{t r}$ has been found only implicitly, as the solution of a certain non-linear equation relating its value to the system parameters. This equation simplifies considerably in the limit of small or large particles diffusivity, in which two cases the tracer velocity is calculated explicitly. Further on, we have found that the density profile around the tracer becomes strongly inhomogeneous: the local density of the monolayer particles in front of the tracer is higher than the average and approaches the average value as an exponential function of the distance from the tracer. The characteristic length and the amplitude of the density relaxation function are calculated explicitly. On the other hand, past the tracer the local density is lower than the average; we show that depending on the condition whether the number of particles in the monolayer is explicitly conserved or not, the local density past the tracer may tend to the average value either as an exponential or as an algebraic function of the distance, revealing in the latter case especially strong memory effects and strong correlations between the particle distribution in the monolayer and the tracer position.

\section{Acknowledgments.}

The authors wish to thank J.De Coninck, A.Lemarchand and A.A.Ovchinnikov for helpful discussions. This work was supported in part by the French-German collaborative research program PROCOPE.

\section{Appendix 1: Series representation of $F_{n, m}$, Eq. (31), in the limit (a)}

We calculate to the first order in powers of $D_{0} \tau^{*} / \sigma^{2}$ the functions $F_{n, m}$ involved in Eq.(39):

$$
F_{0,0}=1+2 \frac{V^{(0)} \sigma}{\tau^{*}\left(V^{(0)}+\sigma(f+g) / \tau^{*}\right)^{2}} \frac{D_{0} \tau^{*}}{\sigma^{2}}+\mathcal{O}\left(\left(\frac{D_{0} \tau^{*}}{\sigma^{2}}\right)^{2}\right),
$$




$$
\begin{gathered}
F_{1,0}=F_{0,1}=\frac{\sigma}{\tau^{*}\left(V^{(0)}+\sigma(f+g) / \tau^{*}\right)} \frac{D_{0} \tau^{*}}{\sigma^{2}}+\mathcal{O}\left(\left(\frac{D_{0} \tau^{*}}{\sigma^{2}}\right)^{2}\right), \\
F_{-1, \pm 1}=2 \frac{V^{(0)} \sigma}{\tau^{*}\left(V^{(0)}+\sigma(f+g) / \tau^{*}\right)^{2}} \frac{D_{0} \tau^{*}}{\sigma^{2}}+\mathcal{O}\left(\left(\frac{D_{0} \tau^{*}}{\sigma^{2}}\right)^{2}\right), \\
F_{-1,0}=\frac{(f+g) \sigma^{2}\left((f+g)\left(V^{(1)}+\sigma / \tau^{*}\right)+V^{(0)}\left(V^{(1)} \tau^{*} / \sigma-2\right)\right)}{\left(V^{(0)}+\sigma(f+g) / \tau^{*}\right)} \frac{D_{0} \tau^{*}}{\sigma^{2}}+\mathcal{O}\left(\left(\frac{D_{0} \tau^{*}}{\sigma^{2}}\right)^{2}\right),
\end{gathered}
$$

and

$$
\begin{aligned}
F_{-2,0} & =\frac{V^{(0) 2}}{\left(V^{(0)}+\sigma(f+g) / \tau^{*}\right)^{2}}+ \\
& +2 \frac{\sigma V^{(0)}\left((f+g)^{2}\left(V^{(1)} \sigma / \tau^{*}+\left(\sigma / \tau^{*}\right)^{2}\right)+(f+g) V^{(0)}\left(V^{(1)}-2 \sigma / \tau^{*}\right)-V^{(0) 2}\right)}{\tau^{*}\left(V^{(0)}+\sigma(f+g) / \tau^{*}\right)^{4}} \frac{D_{0} \tau^{*}}{\sigma^{2}}+ \\
& +\mathcal{O}\left(\left(\frac{D_{0} \tau^{*}}{\sigma^{2}}\right)^{2}\right),
\end{aligned}
$$

while

$$
F_{2,0}=F_{1, \pm 1}=F_{0,2}=\mathcal{O}\left(\left(\frac{D_{0} \tau^{*}}{\sigma^{2}}\right)^{2}\right)
$$

\section{Appendix 2: Explicit results for the amplitudes and the decay lengths of the density profiles at large distances}

\subsection{In front of the tracer}

We find the following explicit asymptotical forms for the decay length and the amplitude:

(a) For small $D_{0}, \delta>0$ and $\mathcal{P} \gg 1$,

$$
\lambda_{+} \sim \ln ^{-1}\left(\frac{\sigma^{2}(f+g)\left(1+(f+g) \tau / \tau^{*}\right)}{D_{0} \tau^{*}\left(\rho+(f+g) \tau / \tau^{*}\right)}\right),
$$

and

$$
K_{+} \sim \frac{\rho_{L}\left(1-\rho_{L}\right)(f+g)^{3 / 2}}{2 \sqrt{\pi}} \frac{\left(1+(f+g) \tau / \tau^{*}\right)^{3 / 2}}{\left(\rho_{L}+(f+g) \tau / \tau^{*}\right)^{5 / 2}}\left(\frac{\sigma^{2}}{D_{0} \tau^{*}}\right)^{3 / 2},
$$

which means that $\lambda_{+}$is logarithmically small with $D_{0}$, while $K_{+}$is large. Note also that $K_{+}$can be a non-monotonous function of $\rho_{L}$ when $(f+g) \tau / \tau^{*} \gg \rho_{L}$.

(b) For small $D_{0}, \delta=0$ and $\mathcal{P} \gg 1$,

$$
\lambda_{+} \sim \frac{1}{\ln \mathcal{P}}=\ln ^{-1}\left(\frac{2\left(1-\rho_{L}\right.}{\rho_{L}}\right),
$$


and

$$
K_{+} \sim \frac{\mathcal{P}^{3 / 2}}{4 \sqrt{2} \pi}
$$

i.e., $\lambda_{+}$is logarithmically small with $\mathcal{P}$, while $K_{+}$is large.

(c) For small $D_{0}, \delta=0$ and $\mathcal{P} \ll 1$,

$$
\lambda_{+} \sim 1 / \mathcal{P}=\frac{\alpha \rho_{L}}{1-\rho_{L}}
$$

and

$$
K_{+} \sim \frac{1}{\pi}(1+\alpha) \rho_{L} \mathcal{P}^{3 / 2}=\frac{1}{\pi}(1+\alpha) \frac{\left(1-\rho_{L}\right)^{3 / 2}}{\alpha^{3 / 2} \rho_{L}^{1 / 2}}
$$

Hence, in this case $\lambda_{+}$is large, since it is inversely proportional to the small parameter $\mathcal{P}$, but $K_{+}$is small.

(d) For $D_{0} \rightarrow \infty$ and $\delta>0$,

$$
\lambda_{+} \sim \frac{1}{2 \sqrt{\delta}}=\sigma^{-1} \sqrt{\frac{D_{0} \tau^{*}}{(f+g)}}
$$

and

$$
K_{+} \sim(1+\alpha) \rho_{L} \mathcal{P}\left(\delta / \pi^{2}\right)^{1 / 4}=\frac{(1+\alpha) \rho_{L}\left(1-\rho_{L}\right) \sigma^{2}}{\sqrt{\pi} D_{0} \tau}\left(\frac{\sigma^{2}(f+g)}{4 D_{0} \tau^{*}}\right)^{1 / 4},
$$

i.e., here, likewise to the case (c), $\lambda_{+}$appears to be large and $K_{+}$is small. On the other hand, if we suppose that $\delta=0$ (no adsorption/desorption) and $D_{0} \rightarrow \infty$, we find a bit different forms of $K_{+}$and $\lambda_{+}$; namely,

$$
\lambda_{+} \sim \frac{1}{\mathcal{P}}=\frac{D_{0} \tau}{\left(1-\rho_{L}\right) \sigma^{2}}
$$

and

$$
K_{+} \sim \frac{(1+\alpha) \rho_{L} \mathcal{P}^{3 / 2}}{\sqrt{\pi}}=\frac{(1+\alpha) \rho_{L}}{\sqrt{\pi}}\left(\frac{\sigma^{2}\left(1-\rho_{L}\right)}{D_{0} \tau}\right)^{3 / 2}
$$

i.e., similarly to the above considered case, $\lambda_{+}$is large and $K_{+}$is small. Note also that, despite the fact that the tracer velocity in the cases $\delta>0$ and $\delta=0$ is given by the same expression in Eq.(69), the characteristic properties of the density profile appear to be different when $\delta$ is zero or $\delta \neq 0$.

\subsection{Past the tracer}

A. Non-conserved particles number.

Consider next the asymptotical behavior of $K_{-}$and $\lambda_{-}$in the limit of slow and fast particles diffusion.

For small $D_{0}, \delta>0$ and $\mathcal{P} \gg 1$, which corresponds to the limit (a) in our previous notations,

$$
\lambda_{-} \sim-\ln ^{-1}\left(\frac{\left(1-\rho_{L}\right)}{1+(f+g) \tau / \tau^{*}}\right)
$$


and

$$
K_{-} \sim-\frac{\rho_{L}}{2 \sqrt{\pi}}\left(\frac{(f+g)\left(1+(f+g) \tau / \tau^{*}\right)}{\rho_{L}+(f+g) \tau / \tau^{*}}\left(\frac{\sigma^{2}}{D_{0} \tau^{*}}\right)\right)^{1 / 2}
$$

i.e. $\lambda_{-}$tends to a constant value when $D_{0} \rightarrow 0$, which means that here $\lambda_{-}>\lambda_{+}$, while $\left|K_{-}\right| \propto$ $D_{0}^{-1 / 2}$ diverges when $D_{0} \rightarrow 0$. Note, however, that we evidently have $\left|K_{-}\right| \ll K_{+}$. This means that in the limit (a) the density profiles around the tracer are strongly asymmetric; in front of the tracer we have a condensed, "traffic"-jam-like region, characterized by a high amplitude but of a relatively short spatial extent, while past the tracer there is a depleted region which extends on much longer scales but has a considerably smaller amplitude.

Next, for high particles diffusivity and $\delta>0$, which corresponds to the limit (d), one encounters an exactly opposite situation. Here the values of the characteristic lengths and the amplitudes of the condensed and the depleted regions almost coincide, i.e. $K_{-} \approx-K_{+}$and $\lambda_{-} \approx \lambda_{+}$, where $K_{+}$ and $\lambda_{+}$are given by Eqs. (108) and (107).

\section{B. Conserved particles number.}

Consider next the asymptotic forms of the decay amplitudes in Eq. (88). Using the results of the previous section, we find then the following asymptotical results:

In the limit of small $D_{0}, \delta=0$ and $\mathcal{P} \gg 1$, which corresponds to the limit (b) of Section 5 ,

$$
K_{-} \sim \frac{\mathcal{P}^{1 / 2}}{4 \sqrt{\pi}}=\left(\frac{\left(1-\rho_{L}\right)}{8 \pi \rho_{L}}\right)^{1 / 2}
$$

which means that the amplitude is large in this limit, since $\rho_{L} \ll 1$.

In the limit of small $D_{0}, \delta=0$ and $\mathcal{P} \ll 1$, (limit (c)),

$$
K_{-} \sim \frac{(1+\alpha)}{2}\left(\frac{\rho_{L}\left(1-\rho_{L}\right)}{\pi \alpha}\right)^{1 / 2}
$$

Since this limit can be only realized at sufficiently high particles densities, $\rho_{L} \sim 1$, we have then that in this case the amplitude should be small.

Lastly, in the limit $D_{0} \rightarrow \infty$ (limit (d)) we find

$$
K_{-} \sim \frac{(1+\alpha) \rho_{L}}{2}\left(\frac{\left(1-\rho_{l}\right) \sigma^{2}}{\pi D_{0} \tau}\right)^{1 / 2}
$$

i.e. which signifies that in this case the decay amplitude is small.

\section{References}

[1] M.-C.Desjonquéres and D.Spanjaard, Concepts in Surface Physics, (Springer Verlag, Berlin, 1996)

[2] D.J.Shaw, Introduction to Colloid and Surface Chemistry, (Butterworth-Heinemann Ltd., Oxford, 1992)

[3] R.H.Fowler and E.A.Guggenheim, Statistical Thermodynamics, (Cambridge University Press, London, 1939)

[4] S.F.Burlatsky, G.Oshanin, A.M.Cazabat and M.Moreau, Phys. Rev. Lett. 76, 86 (1996)

[5] S.F.Burlatsky, G.Oshanin, A.M.Cazabat, M.Moreau and W.P.Reinhardt, Phys. Rev. E 54, $3892(1996)$ 
[6] D.Ausserré, F.Brochard-Wyart and P.G.de Gennes, C. R. Acad. Sci. Paris 320, 131 (1995)

[7] B.N.J.Persson and E.Tosatti, Phys. Rev. B 50, 5590 (1994)

[8] G.Oshanin, J.De Coninck, A.M.Cazabat and M.Moreau, Phys. Rev. E 58, R20 (1998)

[9] G.Oshanin, J.De Coninck, A.M.Cazabat and M.Moreau, J. Mol. Liquids 76, 195 (1998)

[10] see, e.g. H.Jeong, B.Kahng and D.E.Wolf, Physica A 245, 355 (1997); J.G.Amar and F.Family, Phys. Rev. Lett. 74, 2066 (1995) and references therein

[11] R.Gomer, Rep. Prog. Phys. 53, 917 (1990)

[12] H.J.Kreuzer, in: Diffusion at Interfaces: Microscopic Concepts, Springer Series in Surface Science, Vol. 12 (Springer-Verlag, Berlin, 1986)

[13] M.A.Zaluska-Kotur and L.A.Turski, Physica A 195, 375 (1993); Z.W.Gortel, M.A.ZaluskaKotur and L.A.Turski, Phys. Rev. B 52, 16916 (1995)

[14] R.Ferrando, R.Spadacini and G.E.Tommei, Phys. Rev. E 48, 2437 (1993) and references therein

[15] K.W.Kehr and K.Binder, in: Application of the Monte Carlo Method in Statistical Physics, ed. K.Binder, (Springer-Verlag, Berlin, 1987) and references therein.

[16] K.Nakazato and K.Kitahara, Prog. Theor. Phys. 64, 2261 (1980)

[17] R.A.Tahir-Kheli and R.J.Elliott, Phys. Rev. B 27, 844 (1983)

[18] H.van Beijeren and R.Kutner, Phys. Rev. Lett. 55, 238 (1985)

[19] M.Brummelhuis and H.Hilhorst, Physica A 156, 575 (1989)

[20] J.De Coninck, G.Oshanin and M.Moreau, Europhys. Lett. 38, 527 (1997)

[21] O.Bénichou, A.M.Cazabat, A.Lemarchand, M.Moreau and G.Oshanin, cond-mat/9810163

[22] O.Bénichou, A.M.Cazabat, M.Moreau and G.Oshanin, in preparation

[23] S.F.Burlatsky, G.Oshanin, A.Mogutov and M.Moreau, Phys. Lett. A 166, 230 (1992); S.F.Burlatsky, G.Oshanin, M.Moreau and W.P.Reinhardt, Phys. Rev. E 54, 3165 (1996)

[24] E.Raphael and P.G.de Gennes, Europhys. Lett. 31, 293 (1995)

[25] E.Raphael and P.G.de Gennes, Phys. Rev. E 53, 3448 (1996)

[26] C.Landim, S.Olla and S.B.Volchan, Commun. Math. Phys. 192, 287 (1998)

[27] H.S.Wilf, Generatingfunctionology, (Academic Press, New York, 1990)

[28] E.Montroll and G.Weiss, J. Math. Phys. 6, 167 (1965)

[29] B.D.Hughes, Random Walks and Random Environments, (Oxford Science Publ., Oxford, 1995)

[30] W.McCrea and F.Whipple, Proc. R. Soc. Edinb. 60, 281 (1940); see also F.Spitzer, Principles of Random Walk, (Van Nostrand, Princeton, New Jersey, 1964)

[31] P.Flajolet and A.M.Odlyzko, SIAM Journal on Discrete Math. 3, 216 (1990) 


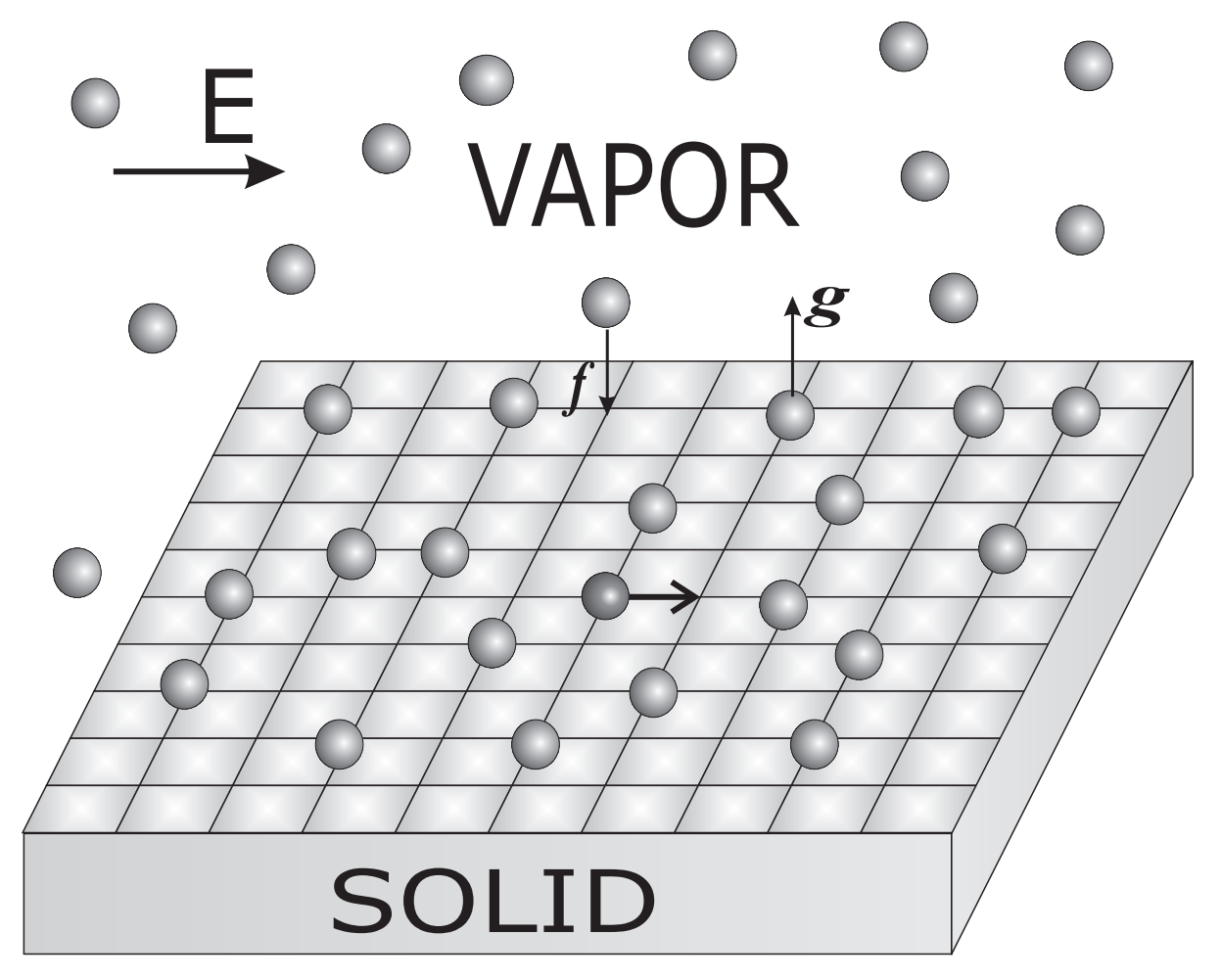

Fig.1. Two-dimensional lattice of adsorption sites partially occupied by identical, mobile hard-core particles (grey spheres) undergoing continuous exchanges with the reservoir - the vapor phase. Particles desorption and adsorption probabilities are denoted by $g$ and $f$, respectively. The dark grey sphere with an arrow denotes the tracer particle, whose motion is completely directed by external field $E$. 


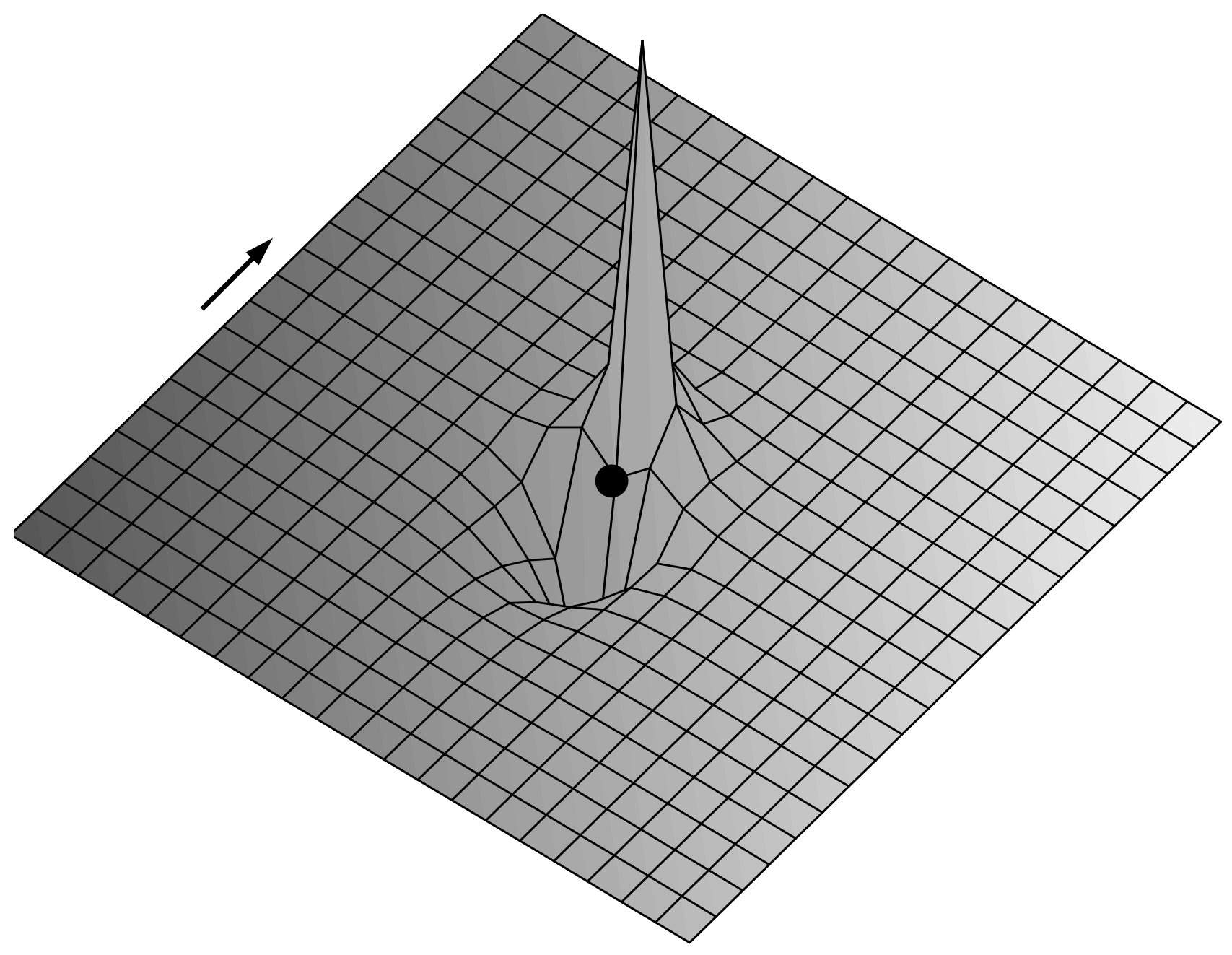

Fig.2a. Sketch of the density profiles at finite distances from the tracer. The black circle denotes the tracer particle and the arrow represents the direction of the external force exerted on the tracer. Limit (a): $D_{0} \rightarrow 0, \delta \gg 1$, and $\mathcal{P} \gg 1$. 


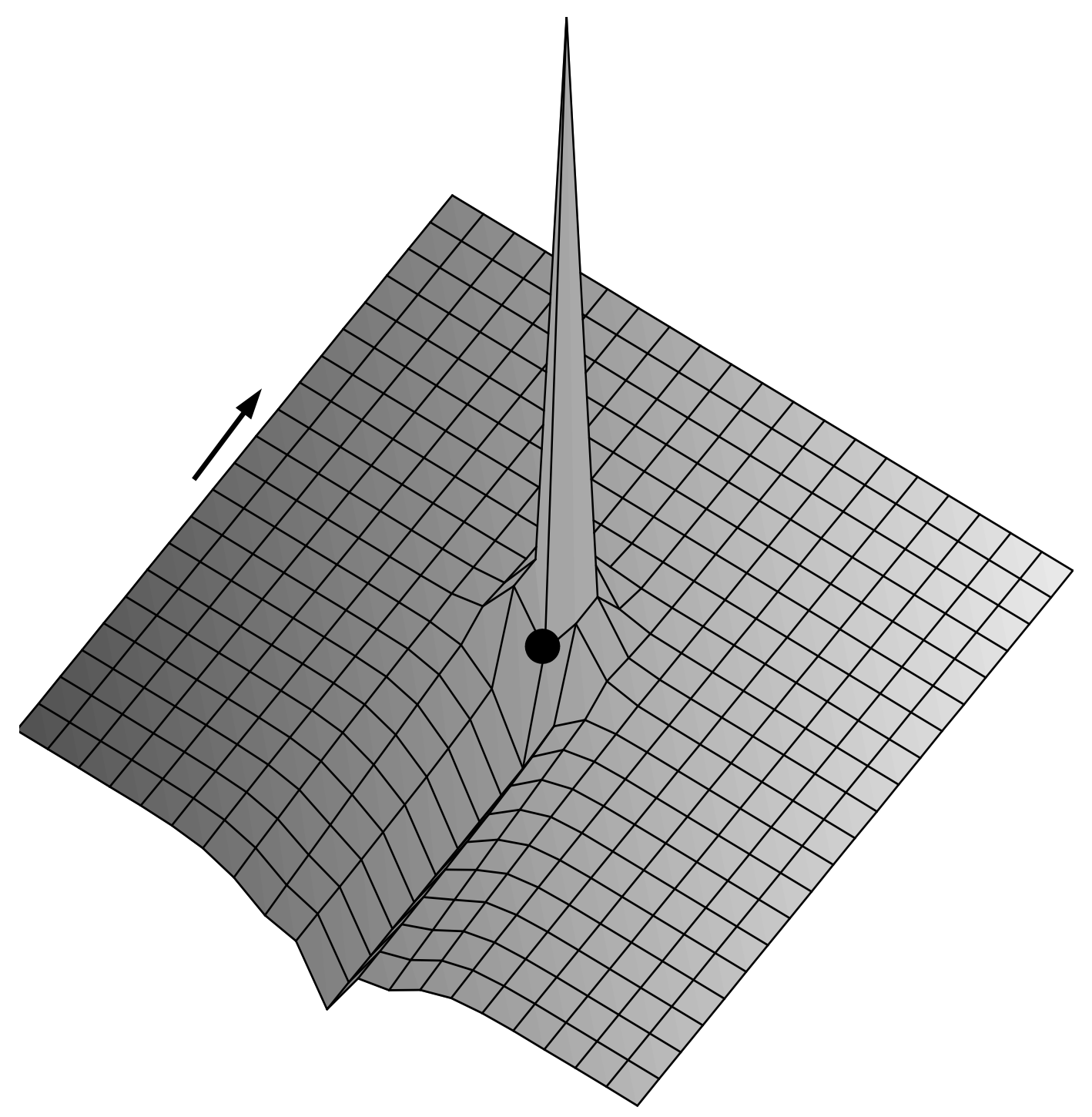

Fig.2b. Sketch of the density profiles at finite distances from the tracer. Limit (b): $D_{0} \rightarrow 0, \delta=0$, and $\mathcal{P} \gg 1$. 


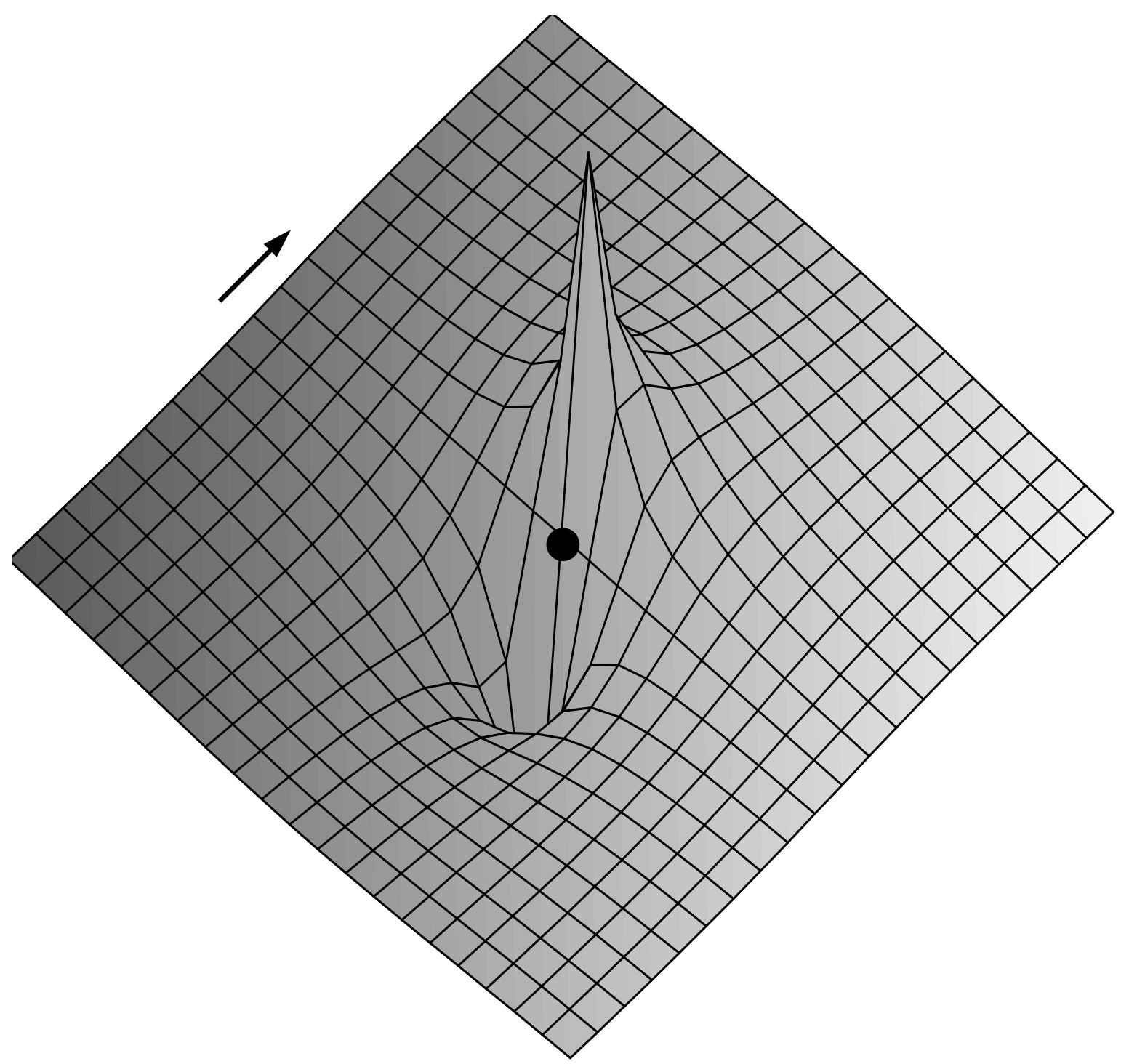

Fig.2c. Sketch of the density profiles at finite distances from the tracer Limit (c): $D_{0} \rightarrow 0, \delta=0$, and $\mathcal{P} \ll 1$. 